\title{
Chemogenetic Recruitment of Specific Interneurons Suppresses Seizure Activity
}

\author{
Alexandru Călin', Mihai Stancu², Ana-Maria Zagrean², John G. R. Jefferys' ${ }^{1}$, \\ Andrei S. Ilie ${ }^{1+}$ and Colin J. Akerman ${ }^{1 * \dagger}$ \\ ${ }^{1}$ Department of Pharmacology, University of Oxford, Oxford, United Kingdom, ${ }^{2}$ Division of Physiology and Neuroscience, \\ Department of Functional Sciences, Carol Davila University of Medicine and Pharmacy, Bucharest, Romania
}

OPEN ACCESS

Edited by:

Laura Cancedda,

Fondazione Istituto Italiano di

Tecnologia, Italy

Reviewed by:

Gabriele Losi,

Istituto di Neuroscienze (IN), Italy

Robert S. Sloviter,

Morehouse School of Medicine,

United States

*Correspondence:

Colin J. Akerman

colin.akerman@pharm.ox.ac.uk

†These authors have contributed equally to this work

Received: 17 July 2018

Accepted: 16 August 2018

Published: 05 September 2018

Citation:

Călin A, Stancu M, Zagrean A-M Jefferys JGR, Ilie AS and Akerman CJ

(2018) Chemogenetic Recruitment of Specific Interneurons Suppresses

Seizure Activity.

Front. Cell. Neurosci. 12:293.

doi: 10.3389/fncel.2018.00293
Current anti-epileptic medications that boost synaptic inhibition are effective in reducing several types of epileptic seizure activity. Nevertheless, these drugs can generate significant side-effects and even paradoxical responses due to the broad nature of their action. Recently developed chemogenetic techniques provide the opportunity to pharmacologically recruit endogenous inhibitory mechanisms in a selective and circuitspecific manner. Here, we use chemogenetics to assess the potential of suppressing epileptiform activity by enhancing the synaptic output from three major interneuron populations in the rodent hippocampus: parvalbumin (PV), somatostatin (SST), and vasoactive intestinal peptide (VIP) expressing interneurons. To target different neuronal populations, promoter-specific cre-recombinase mice were combined with viralmediated delivery of chemogenetic constructs. Targeted electrophysiological recordings were then conducted in an in vitro model of chronic, drug-resistant epilepsy. In addition, behavioral video-scoring was performed in an in vivo model of acutely triggered seizure activity. Pre-synaptic and post-synaptic whole cell recordings in brain slices revealed that each of the three interneuron types increase their firing rate and synaptic output following chemogenetic activation. However, the interneuron populations exhibited different effects on epileptiform discharges. Recruiting VIP interneurons did not change the total duration of epileptiform discharges. In contrast, recruiting SST or PV interneurons produced robust suppression of epileptiform synchronization. PV interneurons exhibited the strongest effect per cell, eliciting at least a fivefold greater reduction in epileptiform activity than the other cell types. Consistent with this, we found that in vivo chemogenetic recruitment of PV interneurons suppressed convulsive behaviors by more than $80 \%$. Our findings support the idea that selective chemogenetic enhancement of inhibitory synaptic pathways offers potential as an anti-seizure strategy.

Keywords: epilepsy, seizure, parvalbumin, somatostatin, vasoactive intestinal peptide, DREADDs, $\mathrm{hM}_{3} \mathrm{D}_{\mathrm{q}}, \mathrm{CNO}$

\section{INTRODUCTION}

Drugs that enhance GABA-mediated synaptic inhibition can be potent anticonvulsants (Czapiński et al., 2005). However, because of their system-wide actions, these drugs exhibit multiple deleterious side-effects (Snodgrass, 1992; Mula, 2011). While GABAergic signaling can become altered in cells within the epileptic focus (Cohen et al., 2002; Huberfeld et al., 2007), inhibitory mechanisms remain effective within the 'penumbra' surrounding the epileptic focus and are able to oppose 
seizure spread (Trevelyan et al., 2006, 2007; Schevon et al., 2012; Cammarota et al., 2013). Selectively enhancing these endogenous inhibitory mechanisms therefore offers the potential to disrupt the propagation of epileptic discharges.

The region of the brain that often contains the epileptic focus in temporal lobe epilepsy patients - the hippocampus - includes multiple subtypes of GABA-releasing interneurons, which are thought to vary in terms of their inhibitory capacity (Klausberger et al., 2003). For instance, because of their intrinsic properties and perisomatic targeting of multiple postsynaptic pyramidal neurons, parvalbumin-expressing (PV) interneurons have been considered particularly effective at inhibiting principal neurons (Cobb et al., 1995; Freund and Buzsáki, 1996; Miles et al., 1996) and at restricting the propagation of network activity (Trevelyan et al., 2006; Cammarota et al., 2013). Meanwhile, because of their post-synaptic targeting, somatostatin-expressing (SST) interneurons have been associated with the regulation of dendritic excitability (Miles et al., 1996; Paz and Huguenard, 2015), which can then affect the spiking output of principal neurons (Lovett-Barron et al., 2012). Other interneuron subtypes, such as vasoactive intestinal polypeptide-expressing (VIP) interneurons, can mediate disinhibitory effects as well as inhibitory effects, apparently because many of their postsynaptic targets are interneurons (Acsády et al., 1996).

Consistent with these ideas, studies using optogenetic strategies to increase interneuron activity have reported promising results in terms of reducing seizure activity (KrookMagnuson et al., 2013; Ledri et al., 2014) and have indicated that interneuron subtypes can exert differential effects upon seizure generation and progression (Cammarota et al., 2013; Krook-Magnuson et al., 2013; Sessolo et al., 2015; Khoshkhoo et al., 2017). However, the temporally synchronous nature of optical activation can also generate counterintuitive effects, as the simultaneous recruitment of interneurons can enhance network synchronization and actually initiate epileptiform activity (Sessolo et al., 2015; Yekhlef et al., 2015; Chang et al., 2018).

A novel alternative strategy is afforded by chemogenetic tools such as Designer Receptors Exclusively Activated by Designer Drugs (DREADDs), which use pharmacological agents to enhance or inhibit the activity of defined cell populations (Armbruster et al., 2007; Alexander et al., 2009). DREADDs are mutated human muscarinic receptors that can be expressed in a cell-specific manner and are not activated by endogenous ligands, but are activated by drugs such as clozapine $\mathrm{N}$-oxide (CNO) and related metabolites (Gomez et al., 2017). Activating excitatory DREADDs, such as the human type-3 muscarinic designer receptor coupled with the $\mathrm{G}_{\mathrm{q}}$ protein $\left(\mathrm{hM}_{3} \mathrm{D}_{\mathrm{q}}\right.$ receptor), is thought to enhance neuronal excitability by downregulating ion channels that hyperpolarize the membrane (Alexander et al., 2009).

Excitatory $\mathrm{hM}_{3} \mathrm{D}_{\mathrm{q}}$ DREADDs have been used to activate interneurons in several brain regions (Hamm and Yuste, 2016; Chen et al., 2017; Wang et al., 2017). However, it remains unclear to what extent different subtypes of GABAergic interneurons can be modulated via DREADDs, and whether chemogenetic control of different interneurons is a viable strategy to reduce epileptiform activity. Here, we demonstrate that three of the major interneuron subtypes in the hippocampus (PV-, SST-, and VIP-expressing interneurons) can be successfully recruited with excitatory DREADDs. The subtypes differ however, in their capacity to increase post-synaptic inhibition in principal neurons and ability to reduce epileptiform synchronization of the neuronal network. By confirming results across in vitro and in vivo models of both chronic and acute seizure activity, our study suggests that chemogenetically enhancing specific interneuron populations may offer an effective anticonvulsant strategy.

\section{MATERIALS AND METHODS}

\section{Preparation and Viral Transduction of Organotypic Hippocampal Brain Slices}

All animal work relating to in vitro preparations was carried out in accordance with the Animals (Scientific Procedures) Act, 1986 (United Kingdom) and under project and personal licenses approved by the Home Office (United Kingdom). Mouse organotypic hippocampal brain slice cultures were prepared from 5- to 7-day-old heterozygous or homozygous, male or female PV-

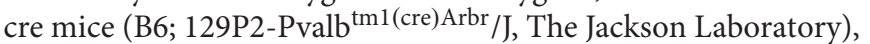
SST-IRES-cre mice (Sst ${ }^{\mathrm{tm} 2.1(\mathrm{cre}) Z \mathrm{j} h} / \mathrm{J}$, The Jackson Laboratory), or

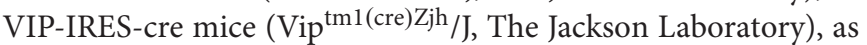
described by Stoppini et al. (1991). All reagents were purchased from Sigma-Aldrich, unless stated otherwise. The brains were extracted and transferred into cold $\left(4^{\circ} \mathrm{C}\right)$ dissection media containing Earle's Balanced Salt Solution $+\mathrm{CaCl}_{2}+\mathrm{MgSO}_{4}$ (Thermo Fisher Scientific), supplemented with $25.5 \mathrm{mM}$ HEPES, $36.5 \mathrm{mM} \mathrm{D}$-glucose, and $5 \mathrm{mM} \mathrm{NaOH}$. The hemispheres were separated, and the individual hippocampi were dissected and immediately sectioned into $400-\mu \mathrm{m}$-thick slices on a McIlwain tissue chopper (Mickle, United Kingdom). Slices were then rinsed in cooled dissection media, placed in 6-well plates onto sterile, porous Millicell-CM membranes, and maintained for 2-8 weeks in culture media containing $78.8 \%$ ( $\mathrm{vol} / \mathrm{vol})$ Minimum Essential Media + GlutaMAX-I (Thermo Fisher Scientific), 20\% (vol/vol) heat-inactivated horse serum (Thermo Fisher Scientific), 1\% (vol/vol) B27 (Thermo Fisher Scientific), $30 \mathrm{mM}$ HEPES, $26 \mathrm{mM}$ D-glucose, $5.8 \mathrm{mM} \mathrm{NaHCO}_{3}, 1 \mathrm{mM} \mathrm{CaCl}_{2}, 2 \mathrm{mM} \mathrm{MgSO}_{4} \cdot 7 \mathrm{H}_{2} \mathrm{O}$, and incubated at $35.5-36^{\circ} \mathrm{C}$ in a $5 \% \mathrm{CO}_{2}$ humidified incubator.

After 3-5 days in culture, organotypic hippocampal slices were transduced with adeno-associated virus (AAV, serotype 8) containing loxP-flanked, inverted DNA sequences under the control of the human Synapsin 1 promoter (University of North Carolina Gene Therapy Center Vector Core and Addgene, United States). Viral DNA contained the doublefloxed sequence for $\mathrm{hM}_{3} \mathrm{D}_{\mathrm{q}}$-mCherry (Addgene \#44361), which was used to target the excitatory DREADDs to specific creexpressing populations. In control experiments and to determine interneuron expression profiles, viral DNA contained the doublefloxed sequence for EGFP (Addgene \#50457). Transduction was achieved by injecting viral particles (mixed with $1 \% \mathrm{wt} / \mathrm{vol}$ fastgreen for visualization) into 5-10 locations along the pyramidal cell layer of the hippocampal slices. Injection pipettes were pulled 
from glass capillaries (1.2 $\mathrm{mm}$ outer diameter, $0.69 \mathrm{~mm}$ inner diameter; Warner Instruments) using a horizontal puller (Sutter P-97), mounted on a manual manipulator (Narishige, Japan) and monitored under a microscope (Leica S6E) coupled with an external fiber optic light source (Photonic Leica CLS 100X). A Picospritzer II system (General Valve) delivered controlled pressure pulses (5-10 psi for $1 \mathrm{~s}$ ) to facilitate gradual diffusion of the viral solution into the tissue. Typical titres were $\sim 10^{12} \mathrm{IU} / \mathrm{ml}$ and injection volumes were $\sim 250 \mathrm{~nL}$ per slice. Feeding media was supplemented with $1 \%(\mathrm{vol} / \mathrm{vol})$ antibiotic and antimycotic solution (with 10,000 units penicillin, $10 \mathrm{mg}$ streptomycin and $25 \mu \mathrm{g}$ amphotericin $\mathrm{B}$ per $\mathrm{mL}$ ) for up to two feeding sessions after injection and slices were allowed at least 2 weeks for expression before being used.

\section{Electrophysiological Recordings in vitro}

The organotypic hippocampal slices were transferred to a recording chamber, where they were maintained at $28^{\circ} \mathrm{C}$ and continuously superfused with artificial cerebrospinal fluid (aCSF) containing (in $\mathrm{mM}$ ): $\mathrm{NaCl}(120), \mathrm{KCl}$ (3), $\mathrm{MgCl}_{2}$ (0.5-1.5), $\mathrm{CaCl}_{2}$ (2-3), $\mathrm{NaH}_{2} \mathrm{PO}_{4}$ (1.2), $\mathrm{NaHCO}_{3}$ (23), D-glucose (11) and ascorbic acid (0.2). Osmolarity was adjusted to $290 \mathrm{mOsm}$ and $\mathrm{pH}$ was adjusted to 7.36 with $\mathrm{NaOH}$. Oxygen and $\mathrm{pH}$ levels were stabilized by bubbling the aCSF with $95 \% \mathrm{O}_{2}$ and $5 \%$ $\mathrm{CO}_{2}$. Neurons within the hippocampal formation were visualized with $10 \times$ and $60 \times$ water-immersion microscope objectives (Olympus BX51WI) and targeted for single or dual-patch wholecell recordings. Patch pipettes of 4-9 M $\Omega$ tip resistance were pulled from filamental borosilicate glass capillaries with an outer diameter of $1.2 \mathrm{~mm}$ and an inner diameter of $0.69 \mathrm{~mm}$ (Warner Instruments), using a horizontal puller (Sutter P-97). For current clamp recordings, pipettes were filled with a potassium-gluconate internal solution (134 mM K-gluconate, $2 \mathrm{mM} \mathrm{NaCl}, 10 \mathrm{mM}$ HEPES, 2 mM Na 2 ATP, 0.3 mM NaGTP, 2 mM MgATP), which had been set to a $\mathrm{pH}$ of 7.36 using $\mathrm{KOH}$, and an osmolarity of $290 \mathrm{mOsm}$. For recording post-synaptic inhibitory currents in voltage clamp, pipettes were filled with a caesium-gluconate internal solution (120 mM Cs-gluconate, $4 \mathrm{mM} \mathrm{NaCl}, 40 \mathrm{mM}$ HEPES, 2 mM MgATP, $0.3 \mathrm{mM}$ NaGTP, and 0.2 mM QX-314). Before use, internal solutions were filtered with a $0.22 \mu \mathrm{m}$ syringe filter (Merck Millipore). Pipettes were mounted to a headstage (CV-7b, Molecular Devices, United States) and controlled via a Multiclamp 700B amplifier (Axon CNS, Molecular Devices). Following entry into whole cell configuration, access resistance $\left(R_{a}\right)$ was monitored every 2 min and experiments were only included if $R_{a}$ remained stable and below $25 \mathrm{M} \Omega$. Recordings were low-pass filtered online at $2 \mathrm{kHz}$ (8-pole Bessel), acquired using Clampex software (pClamp 10, Molecular Devices), and exported into MATLAB (R2017a, Mathworks) for offline analysis using custom-made scripts.

To examine the direct effects of activating excitatory DREADDs upon interneuron excitability, current clamp recordings were conducted in aCSF containing kynurenic acid $(3 \mathrm{mM})$ and $\mathrm{hM}_{3} \mathrm{D}_{\mathrm{q}}$ receptors were activated by bath application of CNO (10-20 $\mu \mathrm{M}$, Tocris, Bio-Techne). The spontaneous action potential firing rate of each interneuron was compared for a 5-min period before and after CNO application, having allowed $3 \mathrm{~min}$ for the $\mathrm{CNO}$ to reach the chamber. To measure the post-synaptic GABAergic currents induced by activating $\mathrm{hM}_{3} \mathrm{D}_{\mathrm{q}}$ receptors in a specific interneuron population, voltage-clamp recordings were conducted by clamping CA1 and CA3 pyramidal neurons at the reversal potential for glutamatergic current ( $\mathrm{E}_{\mathrm{GLUT}}$ ) in the presence of kynurenic acid. Once recordings had stabilized, the amplitude of post-synaptic inhibitory conductances were compared across 2-min periods recorded under baseline conditions, after bath application of $\mathrm{CNO}$ and then after co-administration of $\mathrm{CNO}$ and tetrodotoxin (TTX, 1-2 $\mu \mathrm{M}$ ).

\section{Quantification of Epileptiform Discharges in vitro}

A semi-automated detection algorithm was used to identify the start and end of individual epileptiform discharges (EDs) in vitro. Current-clamp traces were down-sampled to $1 \mathrm{kHz}$ and then band-pass filtered (typically 0.05-0.2 Hz) using a Bessel filter (2nd order). The signal was corrected for the rise time of the filter and subsequently rectified, thresholded and binarized, merging events that were close in time (typically under $1 \mathrm{~min}$ apart), and ignoring events shorter than $5 \mathrm{~s}$. Experiments to test the effects of a drug (e.g., CNO) comprised a 15-min baseline, followed by a 3-min period to allow the drug to reach the recording chamber, and a further 15-min period in which the slice was continuously superfused with drug-containing aCSF. The 15min time periods ('baseline' and 'drug') were assessed using the same ED detection settings. Total ED activity was defined as the sum of time that the slice displayed ED activity during a 15-min period. ED frequency was calculated from the number of EDs that initiated during a 15-min period and ED length was the mean duration of individual EDs that were completely contained within a 15 -min period.

\section{Viral Transduction of Hippocampal Interneurons in vivo}

All animal procedures relating to in vivo experiments were carried out with the approval of the local ethics committee for animal research in Bucharest and in accordance with European Union Directive 2010/63/EU on the protection of animals used for scientific purposes. For viral injections, adult animals of either sex were anesthetized with isoflurane (maintained at 1.5-2\%, $0.4 \mathrm{~L} / \mathrm{min}$ ) and mounted on a stereotaxic instrument (Kopf, RWD Life Science). The level of anesthesia was continuously monitored, eye drops (Corneregel, Bausch and Lomb) were applied to avoid corneal desiccation and a heat pad system (DC Temperature Controller, FHC) was used to maintain the body temperature in the physiological range. Wiretrol II glass capillaries (Drummond Scientific) were pulled using a vertical puller (Narishige PC-10, Japan) and connected to the Hamilton syringe via compression fittings (RN $1 \mathrm{~mm}$, Hamilton, United States). Small craniotomies were generated with a precision drill (FBS 240/E, Proxxon Micromot) and hippocampal bilateral injections were performed both dorsally [-2.18 anteroposterior (AP), 2.3 mediolateral (ML), 2.4-1.85 dorsoventral (DV), relative to Bregma] and ventrally (-2.7 
AP, $2.9 \mathrm{ML}, 3.1-2.25 \mathrm{DV}$, relative to Bregma) at a rate of $1.66 \mathrm{~nL} / \mathrm{s}$, slowly retreating the injection pipette $(0.91 \mu \mathrm{m} / \mathrm{s})$ to maximize delivery throughout the hippocampus. The injection was controlled via a micromanipulator (NeuroCraft MCM, FHC) attached to a syringe (705RN, $50 \mu \mathrm{L}$, Hamilton, United States). Each of four injection tracks was infused with $1,350 \mathrm{~nL}$ of virus (Addgene, United States), of which $170 \mathrm{~nL}$ were delivered at each end of the track. After infusing the target volume of viral solution, a time window of 5 min allowed the virus solution to spread through the hippocampal tissue before the injection pipette was completely retracted. After allowing 2-6 months for expression, and at least 3 days before commencing seizure experiments, mice were anesthetized and implanted with an infusion cannula (C315GS-5 guide cannula, Plastics One, United States) directly over the viral injection site in the right dorsal hippocampus. The cannula was secured to the skull via bone cement (Refobacin R40, Biomet UK, United Kingdom).

\section{Quantification of Seizure Behavior in vivo}

For each seizure experiment, mice were briefly anesthetized with isoflurane to allow insertion of the infusion cannula into the guide cannula, such that the tip of the infusion cannula was located within the hippocampus at $-2.18 \mathrm{AP}, 2.3 \mathrm{ML}, 2.2$ $\mathrm{DV}$, relative to Bregma. At this point, an intraperitoneal (i.p.) bolus of solution containing CNO [ $4 \mathrm{mg} / \mathrm{kg}$ with $4 \%$ dimethyl sulfoxide (DMSO) in saline] or vehicle (4\% DMSO in saline) was administered and the mouse was allowed to fully recover from anesthesia for $15 \mathrm{~min}$ before starting experiments. To monitor behavior, the mouse was placed in a square arena $(400 \mathrm{~mm}$ by $400 \mathrm{~mm}$ ) in which it was able to move freely virtue of a connector assembly (C313C, Plastics One, United States) and swivel system (375/22PS blue, 22ga, Instech, United States), which connected the infusion tubing to a $1 \mu \mathrm{L}$ syringe (7101, Hamilton, United States) controlled by an infusion pump (IVAC P6000, Cardinal Health). Following a 20-min baseline period, 4aminopyridine (4-AP) was infused directly into the hippocampus according to a spaced delivery protocol (three 4-AP infusions, each separated by $12 \mathrm{~min}$ and consisting of a $200 \mathrm{~nL}$ injection of $2 \mathrm{mM} 4$-AP over $2 \mathrm{~min}$ ). Infusions were terminated immediately if the mouse reached the stage of generalized motor convulsions. Throughout each experiment video recordings were performed using two high speed, high definition cameras located at a right angle from each other (Hero 3+ Silver, GoPro, United States) at 60 frames per second, $1920 \times 1080$ pixels per frame. Polarized filters were used to reduce glare from the arena walls. A third camera captured the animals' movements directly from above, allowing to track the location and locomotor activity of the mouse at any given time point. Seizure behavior was blindly scored using the Racine scale. Individual behaviors were considered as binary point events across time at a sampling frequency of $1 \mathrm{~Hz}$. Racine 1 and 2 behaviors were classified as 'nonconvulsive' events, and Racine 3, 4, and 5 behaviors were considered 'convulsive' events (Bergstrom et al., 2013; Tse et al., 2014; Sharma et al., 2018). For the cumulative convulsive Racine index, convulsive events were weighted according to the Racine classification of the behavior: (1) limb clonic activity (Racine 3);
(2) retreating/rearing with orofacial clonic activity (Racine 4); (3) rearing and falling and/or jumping (Racine 5 - full motor convulsions).

\section{Immunohistochemistry and Quantification of Interneuron Distribution}

For in vitro studies, organotypic hippocampal slices expressing $\mathrm{hM}_{3} \mathrm{D}_{\mathrm{q}}$-mCherry or EGFP in specific interneuron populations were fixed overnight at $4^{\circ} \mathrm{C}$ in $4 \%$ paraformaldehyde with $4 \%$ sucrose, in $0.01 \mathrm{M}$ phosphate buffer solution (PBS), $\mathrm{pH}$ 7.4. The slices used for immunofluorescence were washed and embedded in $3 \%$ agar, and re-sectioned at $50 \mu \mathrm{m}$ on a vibrating microtome (Microm HM 650V, Thermo Fisher Scientific). For in vivo studies, mice expressing $\mathrm{hM}_{3} \mathrm{D}_{\mathrm{q}}$-mCherry were transcardially perfused and the brains sectioned at $50 \mu \mathrm{m}$. PV expression was visualized by incubating sections in 1:500 guinea pig primary antibody (cat. no. 195 004, Synaptic Systems) in PBS with $0.3 \%$ Triton-X (PBST) with $1 \%$ normal goat serum (NGS, Thermo Fisher Scientific) overnight at $4^{\circ} \mathrm{C}$, followed by 1:500 Alexa 488 goat anti-guinea pig secondary antibody (Thermo Fisher Scientific) in PBST with 1\% NGS overnight at $4^{\circ} \mathrm{C}$. For SST immunolabeling, the tissue was processed with a basic antigen retrieval kit at $95^{\circ} \mathrm{C}$ for $10 \mathrm{~min}$ (R\&D Systems). All sections were pre-incubated in 10\% NGS in PBST for at least $2 \mathrm{~h}$ at room temperature. SST expression was visualized by incubating sections in 1:250 rat primary antibody (MAB 354, Millipore) in PBST with 1\% NGS for 11 days at $4^{\circ} \mathrm{C}$, followed by 1:500 Alexa 488 goat anti-rat secondary antibody (Thermo Fisher Scientific) in PBST with $1 \%$ NGS for 2 days at $4^{\circ} \mathrm{C}$. VIP expression was visualized by incubating sections in 1:5000 rabbit primary antibody (donation from Professor Peter P. Somogyi) in PBST with 1\% NGS for 2 days at $4^{\circ} \mathrm{C}$, followed by 1:500 Alexa 488 goat anti-rabbit secondary antibody (Thermo Fisher Scientific) in PBST with $1 \%$ NGS overnight at $4^{\circ} \mathrm{C}$. Finally, all sections were mounted in Vectashield (Vector Laboratories) and images were captured with an LSM 880 confocal microscope equipped with $488 \mathrm{~nm}$ and $561 \mathrm{~nm}$ lasers, a $20 \times$ water-immersion objective (W PlanApochromat, NA 1.0) and controlled via the ZEN black software (Zeiss).

To determine the number and distribution of soma and processes associated with each interneuron subtype, we performed quantitative image analysis on organotypic hippocampal slices from PV-cre, SST-cre, and VIP-cre mice that had been injected with floxed AAVs. Slices were subjected to confocal microscopy and, in the resulting images, the CA areas were linearized along the pyramidal layer and the soma of fluorescent neurons were automatically detected via a custommade, two-pass algorithm extracting maximally stable extremal region features using MATLAB (Matas et al., 2004; Nistér and Stewénius, 2008). The number of virally transduced interneurons was derived directly from the number of fluorescent soma per optical section. Meanwhile, to describe the distribution of processes associated with each interneuron subtype, soma were digitally removed from the linearized images to generate a transverse expression profile of fluorescent processes relative to 
the pyramidal cell layer. To compare across multiple interneuron populations, these expression profiles were normalized by the area under each curve.

\section{Data Analysis}

Digital signal processing and presentation were performed using custom-made programs in the MATLAB environment (R2017b, Mathworks). Figures were built using vector-based graphic design in CorelDraw (X6, Corel Corporation) and the statistical software GraphPad Prism (v6.01, GraphPad Software). Video data processing for tracking locomotor activity of animals was performed using the open-source software Bonsai v2.3 (Lopes et al., 2015). Data are presented as mean \pm standard error of mean, and the statistical tests are reported at the relevant points in the text (GraphPad Prism; MATLAB). Nonparametric tests were used when a normal distribution of data could not be ascertained. Appropriate post hoc tests were used when ANOVA tests confirmed a statistically significant effect.

\section{RESULTS}

\section{Recruiting Distinct Hippocampal GABA-Releasing Interneuron Populations With Excitatory DREADDs}

To examine the potential of enhancing the synaptic output of hippocampal interneurons chemogenetically, we used mouse organotypic hippocampal brain slices. This system enabled us to perform targeted patch clamp recordings to determine the pre-synaptic and post-synaptic efficacy of DREADDs, as well as the opportunity to examine the impact of interneuron recruitment upon spontaneously generated epileptiform activity. Organotypic hippocampal brain slices can be used as a model of temporal lobe epilepsy because when kept in culture beyond 2 weeks, they develop spontaneous EDs without any pharmacological treatment, analogous to epileptogenesis in post-traumatic epilepsy (Dyhrfjeld-Johnsen et al., 2010; Lillis et al., 2015). As in previous work (Trevelyan et al., 2006; Sessolo et al., 2015), our recordings from excitatory pyramidal neurons within the Cornu Ammonis (CA) areas revealed reproducible spontaneous EDs that exhibited a sustained duration (mean duration $48.3 \pm 8 \mathrm{~s}$ ) and stable frequency (Figures 1A,B). Consistent with epileptiform activity in many systems (Trevelyan et al., 2006; Sessolo et al., 2015), these spontaneous EDs recruited both excitatory and inhibitory neurons within the network. Paired recordings revealed that during each ED, pyramidal neurons received intense barrages of both glutamatergic and GABAergic post-synaptic currents (Figures 1C,D). To further characterize EDs, we tested the effect of first-line anti-epileptic drugs, valproate and carbamazepine. At therapeutically relevant doses, both drugs were ineffective at suppressing EDs (Figure 2), consistent with the idea that organotypic slices represent a model of drug-resistant temporal lobe epilepsy (Albus et al., 2008; Avaliani et al., 2016). By combining promoter-specific cre-recombinase mice with floxed chemogenetic constructs, we were then able to investigate the cellular and network effects of delivering DREADDs to specific interneuron populations.

Parvalbumin interneurons, a principal GABAergic population, reside primarily within the pyramidal layer of CA areas (Pawelzik et al., 2002), and their axons target the somatic compartment of pyramidal neurons (Pawelzik et al., 2002; Bartos and Elgueta, 2012). Consistent with this, injection of AAV containing floxed constructs into organotypic hippocampal slices from PV-cre mice resulted in somatic and process expression that was restricted to the pyramidal cell layer (Figures 1E,F). Immunohistochemical experiments confirmed that the PV interneurons could be efficiently and specifically targeted with the excitatory DREADD receptor, $\mathrm{hM}_{3} \mathrm{D}_{\mathrm{q}}$. Two to four weeks after viral transduction with $\mathrm{AAV}_{8}-\mathrm{hSyn}-\mathrm{DIO}-$ $\mathrm{hM}_{3} \mathrm{D}_{\mathrm{q}}$-mCherry, the majority of expressing neurons were immunopositive for PV (95.2 $\pm 1.5 \%$ 'specificity') and the majority of all PV immunopositive neurons were expressing $\mathrm{hM}_{3} \mathrm{D}_{\mathrm{q}}$-mCherry (90.5 $\pm 1.4 \%$ 'efficiency'; Figure 1G). To assess whether activating $\mathrm{hM}_{3} \mathrm{D}_{\mathrm{q}}$ receptors could increase the output of the PV interneuron population, current clamp recordings were targeted to $\mathrm{hM}_{3} \mathrm{D}_{\mathrm{q}}$-mCherry positive neurons. The addition of the $\mathrm{hM}_{3} \mathrm{D}_{\mathrm{q}}$ ligand, $\mathrm{CNO}$, led to a significant increase in the firing rate of PV interneurons, from $0.5 \pm 0.3 \mathrm{~Hz}$ during baseline, to $1.9 \pm 1.1 \mathrm{~Hz}$ in the presence of $\mathrm{CNO}$ (Figure 1H).

We performed similar experiments in slices generated from SST-cre mice and VIP-cre mice (Figure 3). Consistent with previous evidence that SST interneurons target the dendritic compartments of hippocampal principal neurons (Katona et al., 1999; Lovett-Barron et al., 2012), we found that the soma and processes of SST interneurons were located within stratum oriens and lacunosum-moleculare, and tended to avoid the pyramidal cell layers (Figures 3A,B). Immunohistochemistry confirmed that the SST interneurons were efficiently $(89.6 \pm 1.5 \%)$ and specifically (94.1 $\pm 1.6 \%$; Figure $3 \mathrm{C}$ ) targeted with $\mathrm{hM}_{3} \mathrm{D}_{\mathrm{q}}$ receptors. Consistent with the results in PV interneurons, CNO significantly increased the firing rate of SST interneurons, from $1.1 \pm 0.5 \mathrm{~Hz}$ during baseline, to $3.7 \pm 1.3 \mathrm{~Hz}$ (Figure 3D).

Consistent with previous evidence that distinct VIP interneuron subtypes target oriens lacunosum-moleculare interneurons and the perisomatic regions or proximal dendrites of pyramidal neurons (Léránth et al., 1984; Acsády et al., 1996; Chamberland et al., 2010; Tyan et al., 2014) (Figure 3E), we found that the soma and processes of virally transduced VIP interneurons were located within stratum radiatum/lacunosummoleculare, pyramidale and oriens in slices from the VIP-cre mice (Köhler, 1982; Taniguchi et al., 2011) (Figure 3F). The majority of expressing neurons were immunopositive for VIP (81.2 $\pm 2.4 \%$ specificity) and the majority of VIP immunopositive neurons expressed the floxed construct (81.6 $\pm 4.4 \%$ efficiency; Figure 3G). Finally, CNO increased the firing rate from $0.01 \pm 0.01 \mathrm{~Hz}$ during baseline, to $0.1 \pm 0.1 \mathrm{~Hz}$ (Figure 3H).

A summary plot for the three interneuron populations confirmed that the expression profiles of PV, SST, and VIP 

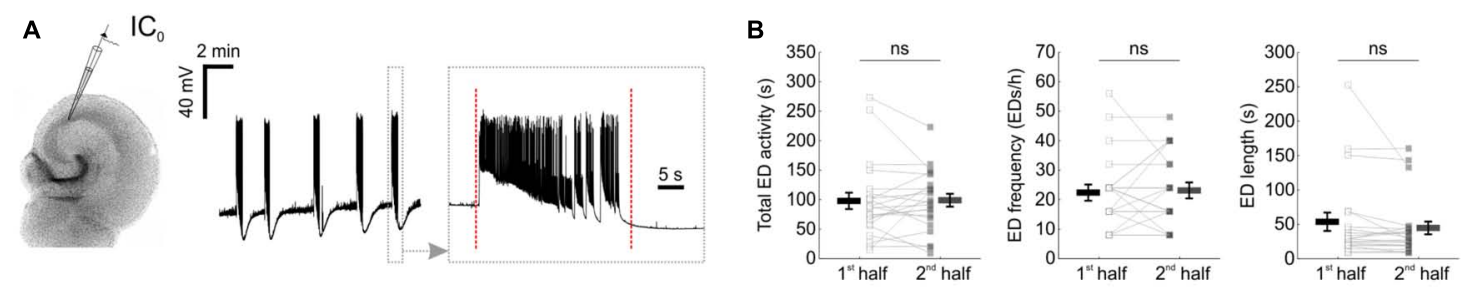

C
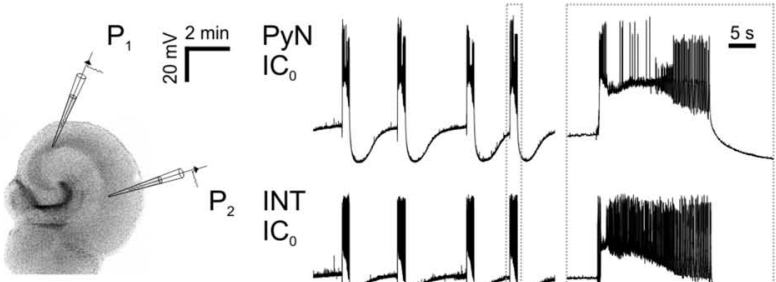

D

$P_{2}$ IN
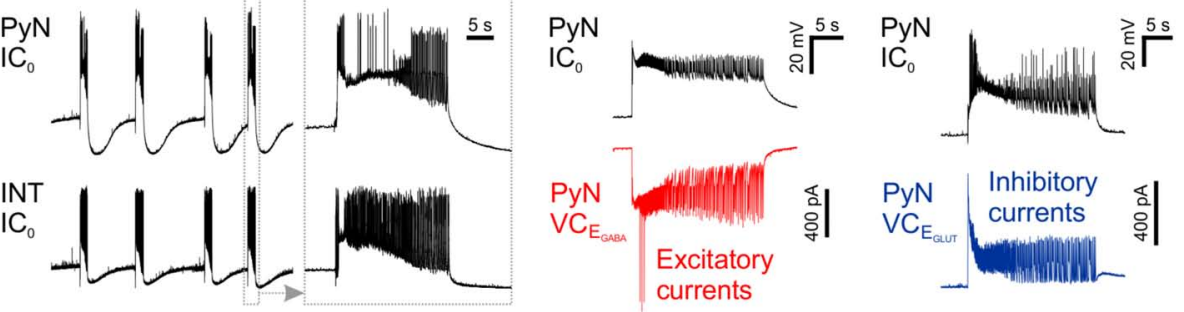

$E$

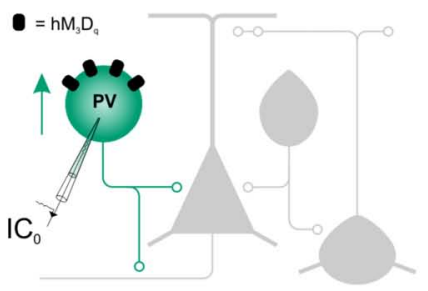

G

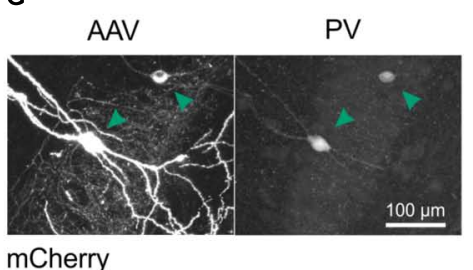

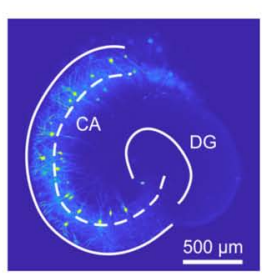

$\mathbf{F}$
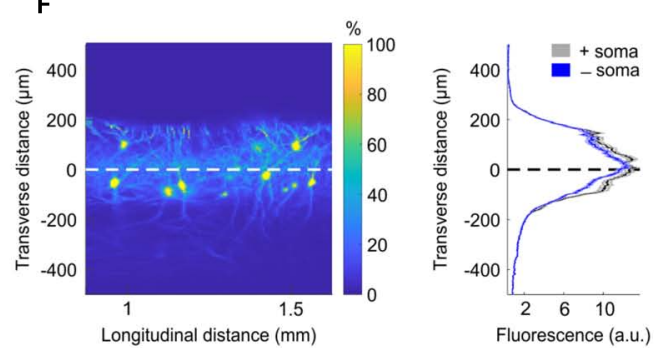

Fluorescence (a.u.)
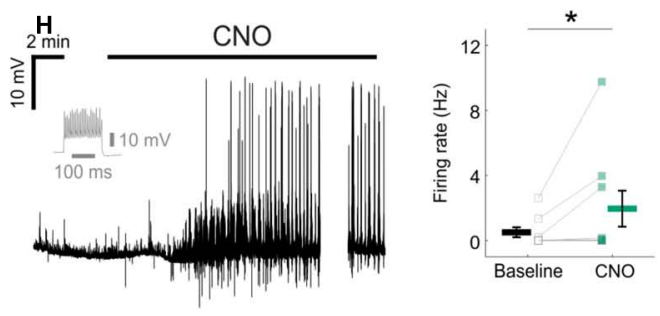

FIGURE 1 | Chemogenetic recruitment of hippocampal interneurons in an in vitro model of epileptiform activity. (A) Current-clamp recording of a CA3 pyramidal neuron from a mouse organotypic hippocampal slice reveals repeated EDs. The vertical red dotted lines in the expanded trace mark the onset and the termination of the underlying ED, as determined by an automated detection algorithm. (B) A 15-min time window was used to assess the stability of the epileptiform activity. Total ED activity, defined as the cumulative duration of ED activity [left, $96.8 \pm 13.9 \mathrm{~s}$ during $1 \mathrm{st}$ half, $97.9 \pm 10.9 \mathrm{~s}$ during $2 \mathrm{nd}$ half; $N=22$ slices, $W_{(21)}=110.5$, $p=0.8715$, two-tailed Wilcoxon signed-rank test], ED frequency [middle, $22.2 \pm 2.7 \mathrm{EDs} / \mathrm{h}$ during 1st half, $22.9 \pm 2.7 \mathrm{EDs} / \mathrm{h}$ during $2 \mathrm{nd}$ half; $N=22$ slices, $W_{(12)}=34, p=0.7153$, two-tailed Wilcoxon signed-rank test], and individual ED length [right, $52.8 \pm 13.4 \mathrm{~s}$ during $1 \mathrm{st}$ half, $43.9 \pm 9.2 \mathrm{~s}$ during $2 \mathrm{nd}$ half; $N=22$ slices, $W_{(21)}=71, p=0.1281$, two-tailed Wilcoxon signed-rank test] were stable across the 15-min time window. (C) A dual current-clamp (IC 0 ) electrophysiological recording of a CA3 pyramidal neuron (PyN) and a GABAergic interneuron (INT) reveal spontaneous EDs in a mouse organotypic hippocampal brain slice (left). Expanded traces (right) show that both excitatory and inhibitory neurons are recruited during the EDs. (D) Simultaneous current clamp and voltage clamp (VC) recordings from pairs of pyramidal neurons demonstrate that strong barrages of excitatory (bottom-left, red) and inhibitory (bottom-right, blue) post-synaptic currents occur throughout the EDs, as monitored by current clamp recordings from a neighboring pyramidal neuron (top). $\mathrm{E}_{\mathrm{GABA}}$, reversal potential for GABAergic current; $E_{G L U T}$, reversal potential for glutamatergic current. (E) Cartoon of hippocampal circuitry (left) showing the targeting of the $h M_{3} D_{q}$ receptor to $P V$ interneurons. Confocal image (right) of a hippocampal slice from a PV-cre mouse illustrates the fluorescence distribution profile of virally transduced PV interneurons. The superimposed white dashed line marks the center of the pyramidal cell layer. Continuous white lines indicate the dentate gyrus (DG) and outline of the CA areas (CA). (F) The confocal image was linearized (left) to facilitate the quantification of the transverse expression profile for PV interneurons, relative to the pyramidal cell layer (dashed line at zero). This confirmed that PV interneurons (+soma) and their processes (-soma) were restricted to the pyramidal cell layer (right). (G) The immunohistochemical characterization of $\mathrm{PV}$ interneurons transduced with $\mathrm{AAV}_{8}-\mathrm{hSyn}-\mathrm{DIO}-\mathrm{hM}_{3} \mathrm{D}_{\mathrm{q}}-\mathrm{mCherry}$ demonstrates high targeting specificity and efficiency $\left(\mathrm{N}=14\right.$ sections from six slices). (H) An example current-clamp recording of a $\mathrm{PV}$ interneuron expressing $\mathrm{hM}_{3} \mathrm{D}_{\mathrm{q}}$ receptors shows that CNO promotes action potential firing (left). The firing pattern of the neuron in response to a 500 pA square wave current pulse is shown in gray. The break in the recording reflects a period during which the intrinsic properties of the neuron were monitored. Recordings were conducted in kynurenic acid to isolate the direct effects of $h M_{3} D_{q}$ receptor activation, and to prevent the occurrence of EDs. Population data shows a significant increase in firing rate following $\mathrm{CNO}$ [right; $N=9$ slices, $W_{(7)}=0, p=0.0156$, two-tailed Wilcoxon signed-rank test]. * $p<0.05$. 
A
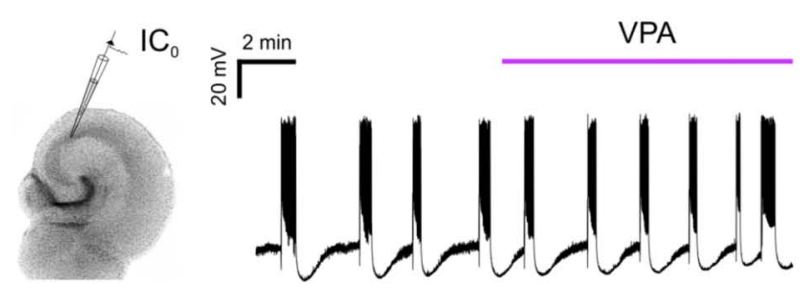

C

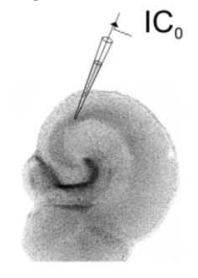

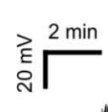

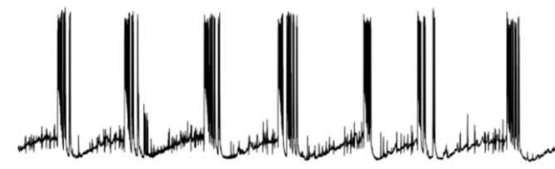

B
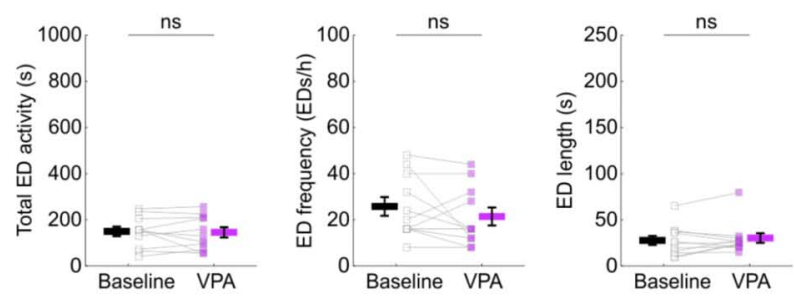

D

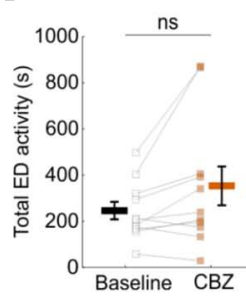

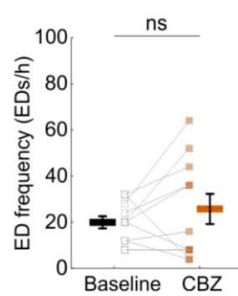

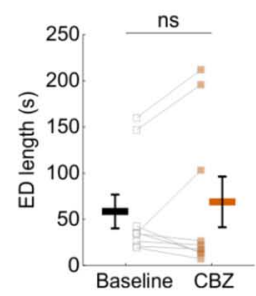

FIGURE 2 | Epileptiform activity in mouse organotypic hippocampal brain slices exhibits resistance to first-line anti-epileptic drugs. (A) A representative recording showing that $1.5 \mathrm{mM}$ valproic acid (VPA) did not disrupt the spontaneous EDs. (B) No impact could be detected of VPA on the total ED activity [left, $146.6 \pm 20.4 \mathrm{~s}$ during baseline and $142.6 \pm 22 \mathrm{~s}$ during VPA; $N=11, t_{(10)}=0.31, p=0.7643$, two-tailed paired $t$-test], frequency [middle, $25.5 \pm 4$ EDs/h during baseline and $21.1 \pm 3.9 \mathrm{EDs} / \mathrm{h}$ during VPA; $N=11, t_{(10)}=1.17, p=0.2674$, two-tailed paired $t$-test] or individual ED length [right, $27 \pm 5 \mathrm{~s}$ during baseline and $29.5 \pm 5.2 \mathrm{~s}$ during VPA; $N=11, W_{(11)}=22, p=0.3652$, two-tailed Wilcoxon signed-rank test]. (C) A representative recording showing that $50 \mu M$ carbamazepine (CBZ) did not disrupt the spontaneous EDs. (D) No impact could be detected of CBZ on the total ED activity [left, $242.7 \pm 37.8 \mathrm{~s}$ during baseline and $350.3 \pm 84.2 \mathrm{~s}$ during CBZ; $N=11, t_{(10)}=2.136, p=0.0584$, two-tailed paired $t$-test], frequency [middle, $19.6 \pm 2.7 \mathrm{EDs} / \mathrm{h}$ during baseline and $25.5 \pm 6.5 \mathrm{EDs} / \mathrm{h}$ during CBZ; $N=11$, $t_{(10)}=1.03, p=0.3266$, two-tailed paired $t$-test] or individual ED length [right, $57.8 \pm 18.3 \mathrm{~s}$ during baseline and $68.1 \pm 27.4 \mathrm{~s}$ during $\mathrm{CBZ} ; \mathrm{N}=9, W_{(9)}=21$, $p=0.9102$, two-tailed Wilcoxon signed-rank test]. VPA and CBZ drug concentrations were equivalent to effective doses in vivo (Albus et al., 2008).

interneurons were significantly different from one another (Figure 3I) and were consistent with data from acute preparations of mouse hippocampus (Taniguchi et al., 2011; Lovett-Barron et al., 2012). These results are in accordance with previous observations that organotypic slices retain fundamental features of the circuit, including the subcellular targeting of perisomatic and dendritic domains of pyramidal neurons by distinct interneuron populations (Streit et al., 1989; De Simoni et al., 2003; Cristo et al., 2004). Meanwhile, CNO-mediated activation of $\mathrm{hM}_{3} \mathrm{D}_{\mathrm{q}}$ receptors resulted in comparable foldincreases in spiking activity across the three interneuron populations: PV interneurons showed a $4.1 \pm 2.4$ fold increase in their firing rate, SST interneurons showed a $3.4 \pm 1.2$ fold increase and VIP interneurons showed a $7.9 \pm 4.4$ fold increase (Figure 3J).

\section{Chemogenetic Enhancement of GABAergic Interneuron Populations Can Attenuate Epileptiform Activity in vitro}

We next tested the effects of enhancing the activity of each interneuron population on EDs. Spontaneous EDs were recorded from pyramidal neurons before and after CNO-mediated activation of the relevant interneuron population. We quantified total ED activity, ED frequency and individual ED duration. In PV-targeted slices (Figure 4A), CNO-mediated activation of $\mathrm{hM}_{3} \mathrm{D}_{\mathrm{q}}$ receptors significantly reduced the total ED activity (from $247.5 \pm 47.8 \mathrm{~s}$ during baseline to $116.5 \pm 47.4 \mathrm{~s}$ during CNO), which resulted from a significant decrease in ED frequency (from 17.6 $\pm 5.3 \mathrm{EDs} / \mathrm{h}$ during baseline to $8.4 \pm 3.3 \mathrm{EDs} / \mathrm{h}$ during $\mathrm{CNO}$ ), without affecting the duration of individual EDs $(65.8 \pm 15.7 \mathrm{~s}$ during baseline and $77.5 \pm 27.4 \mathrm{~s}$ during $\mathrm{CNO}$ ) (Figure 4B). In SST-targeted slices (Figure 4C), CNO also significantly reduced total ED activity (from $263.2 \pm 41.4 \mathrm{~s}$ during baseline to $133.3 \pm 40.2$ $\mathrm{s}$ during $\mathrm{CNO}$ ), which involved a decrease in ED frequency (from $26 \pm 6.8 \mathrm{EDs} / \mathrm{h}$ during baseline to $13.3 \pm 3.5 \mathrm{EDs} / \mathrm{h}$ during $\mathrm{CNO}$ ), without significantly affecting individual ED length $(72 \pm 21.7 \mathrm{~s}$ during baseline and $47.6 \pm 14.5 \mathrm{~s}$ during $\mathrm{CNO}$ ) (Figure 4D). In VIP-targeted slices (Figure 4E), CNO did not decrease the total ED activity $(234.3 \pm 40.6 \mathrm{~s}$ during baseline and $256.3 \pm 62.7 \mathrm{~s}$ during $\mathrm{CNO}$ ). While enhancing VIP interneuron output led to a decrease in ED frequency (from $21.7 \pm 4.3 \mathrm{EDs} / \mathrm{h}$ during baseline to $17.3 \pm 4$ EDs/h during CNO), there was a simultaneous increase in the duration of individual EDs (from $43.5 \pm 10.3 \mathrm{~s}$ during baseline to $69.4 \pm 19.6 \mathrm{~s}$ during $\mathrm{CNO}$ ) (Figure 4F). Finally, to assess potential off-target effects of $\mathrm{CNO}$, the drug was bath-applied during the recording of spontaneous EDs in control slices virally transduced with the same AAV type expressing eGFP in a cre-dependent manner, and not $\mathrm{hM}_{3} \mathrm{D}_{\mathrm{q}}$ (Figure 4G). In these experiments, no changes were detected for total ED activity, ED frequency, or individual ED length (Figure 4H).

Comparisons across the different interneuron populations confirmed subtype-specific effects. Total ED activity was 
A

- $=\mathrm{hM}_{3} \mathrm{D}_{\mathrm{q}}$

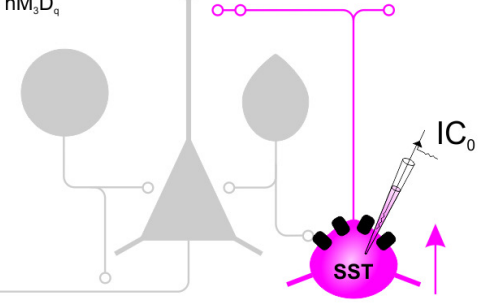

C

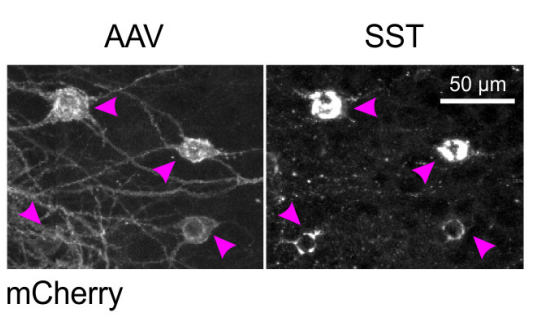

E

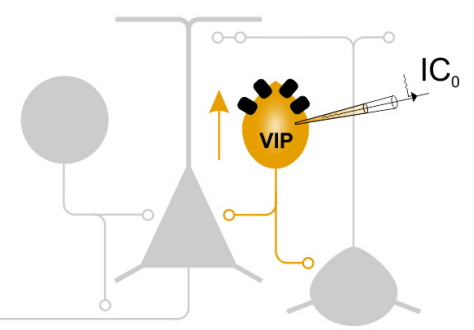

G

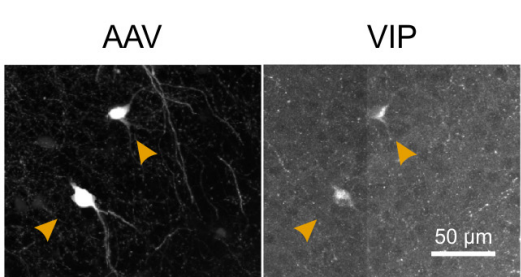

EGFP

I

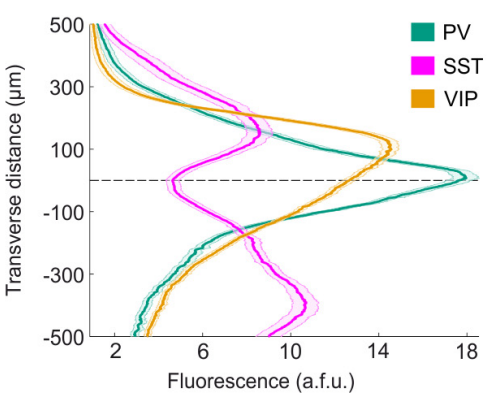

B
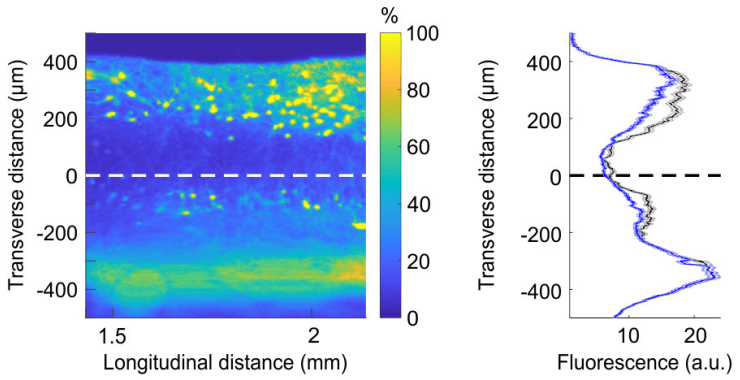

D

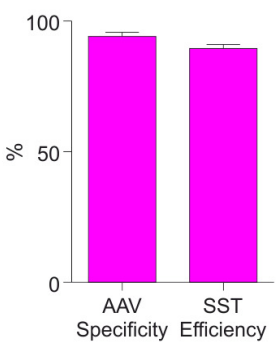

हे

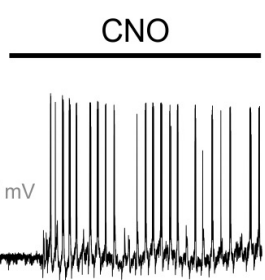

$\mathbf{F}$
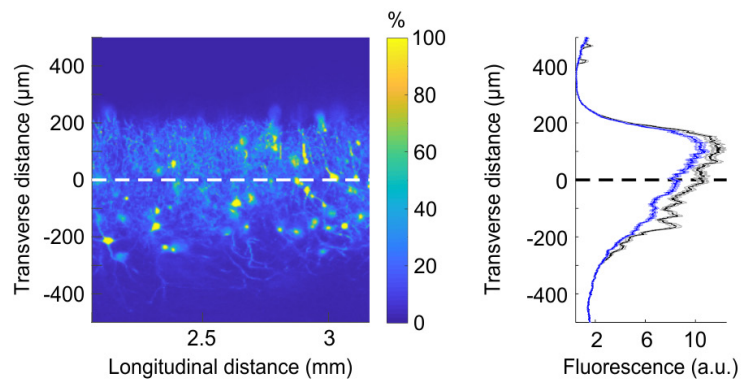

H
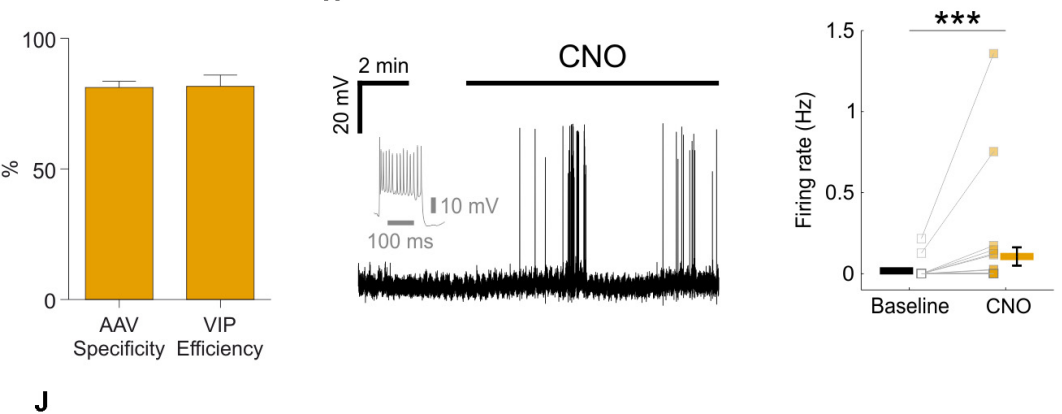

FIGURE 3 | Continued

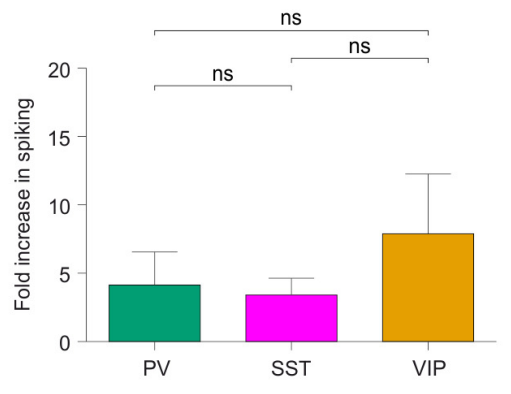




\begin{abstract}
FIGURE 3 | Distinct subtypes of hippocampal GABAergic interneurons can be recruited via excitatory DREADDs. (A) Cartoon (left) showing the targeting of $h M_{3} D_{q}$ receptors to SST hippocampal interneurons. A confocal image of a SST-cre mouse slice (right) illustrates the fluorescence distribution profile of virally transduced SST interneurons. Continuous white lines outline the DG and CA areas. (B) The confocal image was linearized (left) to facilitate quantification of the transverse expression profile for SST interneurons, relative to the pyramidal cell layer (dashed white line at zero). This confirmed that SST interneurons (+soma) and their processes (-soma) were associated with stratum oriens and lacunosum-moleculare (right). (C) Immunohistochemical characterization of SST interneurons transduced with $\mathrm{AAV}_{8}$-hSyn-DIO-hM $\mathrm{D}_{\mathrm{q}}$-mCherry demonstrates high targeting specificity and efficiency ( $N=16$ sections from six slices). (D) Current-clamp recording from a SST interneuron expressing $\mathrm{hM}_{3} \mathrm{D}_{\mathrm{q}}$ in the presence of kynurenic acid, showing that CNO promotes action potential firing (left). The firing pattern of the neuron in response to a $500 \mathrm{pA}$ square wave current pulse is shown in gray. Population data shows a significant increase in firing rate in the presence of CNO [right, $N=13$ slices, $W_{(9)}=4, p=0.0273$, two-tailed Wilcoxon signed-rank test]. (E) Cartoon (left) shows the targeting of $\mathrm{hM}_{3} \mathrm{D}_{\mathrm{q}}$ receptors to VIP interneurons and confocal image (right) illustrates the fluorescence distribution profile of virally transduced interneurons in a VIP-cre slice. (F) Linearizing the confocal image (left) confirmed that the expression profile for VIP interneurons (+soma) and their processes (-soma) was associated with stratum radiatum/lacunosum-moleculare,

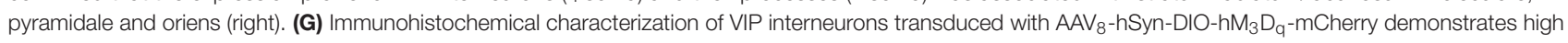
targeting specificity and efficiency ( $N=4$ sections from two slices). (H) Example current-clamp recording from a VIP interneuron expressing $h M_{3} D_{q}$ in $k y n u r e n i c ~ a c i d$, showing that CNO promotes action potential firing (left). The firing pattern of the neuron in response to a 500 pA square wave current pulse is shown in gray. Population data (right) shows a significant increase in firing rate in the presence of CNO $\left[N=27\right.$ slices, $W_{(13)}=1, p=0.0005$, two-tailed Wilcoxon signed-rank test]. (I) Comparison of the fluorescence distribution profiles following viral-transduction of the three interneuron populations [PV, $N=38$ slices; SST, $N=37$ slices; VIP, $N=57$ slices]. Each distribution was normalized by the area under the profile curve and shown to be significantly different between the cell types [interaction between cell type and location: $F_{(8,516)}=58.81 ; p<0.0001$, repeated measures two-way ANOVA]. (J) There was no difference in the CNO-induced fold-increase in spiking rate (normalized to baseline) between the three interneuron populations $\left[\chi^{2}{ }_{(2)}=0.9136, p=0.6333\right.$, Kruskal-Wallis test]. ${ }^{*} p<0.05$, ${ }^{* * *} p<0.001$.
\end{abstract}

reduced by more than half following activation of either PV interneurons (down $58.2 \pm 10.3 \%$ ) or SST interneurons (down $50.8 \pm 10.5 \%$ ), and both of these reductions were significantly greater than the change in total $\mathrm{ED}$ activity induced by recruiting VIP interneurons (up $12.7 \pm 15.4 \%$ ) (Figure 4I). Each of the interneuron populations was able to decrease ED frequency: PV interneurons by $55.4 \pm 10.1 \%$, SST interneurons by $36.7 \pm 13 \%$ and VIP interneurons by $26.3 \pm 10.4 \%$ (Figure 4J). Meanwhile, only the VIP population increased individual ED length by $57.5 \pm 28.4 \%$ (Figure $4 \mathrm{~K}$ ). In summary, chemogenetically enhancing the output of interneuron populations can generate effective suppression of epileptiform synchronization, but the effectiveness of this approach varied by interneuron subtype.

\section{Chemogenetically Enhanced Interneuron Subtypes Differ in Their Post-synaptic Inhibition of Pyramidal Neurons}

To examine the cellular basis of these effects, we performed voltage clamp recordings to compare how chemogenetic recruitment affects the post-synaptic inhibition converging upon pyramidal neurons. Inhibitory post-synaptic currents were isolated at the reversal potential for glutamatergic current ( $\mathrm{E}_{\mathrm{GLUT}}$ ) (Figures 5A,C,E). For each of the three interneuron populations, CNO-activation of $\mathrm{hM}_{3} \mathrm{D}_{\mathrm{q}}$ receptors resulted in a significant increase in post-synaptic inhibitory input (Figures 5A,C,E), which was abolished by bath application of tetrodotoxin (1-2 $\mu \mathrm{M}$; Figures 5A,C,E), confirming that it was mediated by action potential-evoked GABA release. Total inhibitory post-synaptic input to pyramidal neurons increased from $11 \pm 4.2 \mathrm{pA} / \mathrm{ms}$ to $34 \pm 6.1 \mathrm{pA} / \mathrm{ms}$ when PV neurons were recruited, from $9.3 \pm 2.6 \mathrm{pA} / \mathrm{ms}$ to $126.6 \pm 14.6 \mathrm{pA} / \mathrm{ms}$ for SST interneurons and from $5.7 \pm 1.1 \mathrm{pA} / \mathrm{ms}$ to $16.2 \pm 2.4 \mathrm{pA} / \mathrm{ms}$ for VIP interneurons (Figures 5B,D,F). At the population level, the overall increase in post-synaptic inhibitory input resulting from $\mathrm{CNO}$-mediated activation of $\mathrm{hM}_{3} \mathrm{D}_{\mathrm{q}}$ receptors was highest for the SST interneuron population (increase of $116.7 \pm 15.5 \mathrm{pA} / \mathrm{ms}$ ), then the $\mathrm{PV}$ interneuron population (increase of $22.6 \pm 5.7 \mathrm{pA} / \mathrm{ms}$ ), and then the VIP interneuron population (increase of $10.3 \pm 2.3 \mathrm{pA} / \mathrm{ms})$.

To compare across the different subtypes, we expressed our measurements of inhibitory efficacy in terms of individual interneurons. First, the CNO-induced increase in post-synaptic inhibitory input was normalized by the number of $h M_{3} D_{q^{-}}$ expressing cells, as determined from stereological cell counts (Figure 5G; see "Materials and Methods" section). The increase in inhibitory post-synaptic input was similar for an individual $\mathrm{PV}$ interneuron (1.0 \pm 0.3 fold, relative to a PV interneuron) and an SST interneuron $(1.1 \pm 0.1$ fold, relative to a PV interneuron), both of which were five times greater than for an individual VIP interneuron $(0.2 \pm 0.1$ fold, relative to a PV interneuron; Figure 5H). Second, we normalized the effects upon total ED activity by the number of $\mathrm{hM}_{3} \mathrm{D}_{\mathrm{q}}$-expressing cells. Individual PV interneurons were associated with the greatest reduction in epileptiform activity $(1.0 \pm 0.2$ fold, relative to a PV interneuron), which was at least five times more than an individual SST interneuron $(0.2 \pm 0.04$ fold, relative to a PV interneuron) or VIP interneuron $(-0.1 \pm 0.1$ fold, relative to a PV interneuron; Figure 5I). These observations are consistent with the idea that, as a result of their perisomatic targeting and extensive axonal arbors, individual PV interneurons can mediate particularly effective inhibition of pyramidal neurons (Freund and Buzsáki, 1996; Miles et al., 1996).

\section{Chemogenetic Recruitment of PV Interneurons Attenuates Seizure Activity in vivo}

We next assessed the potential of this chemogenetic strategy to reduce seizure activity in vivo. Given our findings in vitro, the $\mathrm{PV}$ interneurons were selected as an effective cell population to target. The $\mathrm{hM}_{3} \mathrm{D}_{\mathrm{q}}$ receptor was delivered to PV hippocampal interneurons by bilateral injections of $\mathrm{AAV}_{8}$-hSyn-DIO$\mathrm{hM}_{3} \mathrm{D}_{\mathrm{q}}$-mCherry in the hippocampus of 4 - to 14 -month-old PV-cre mice (Figure 6A). The virus was delivered at multiple 

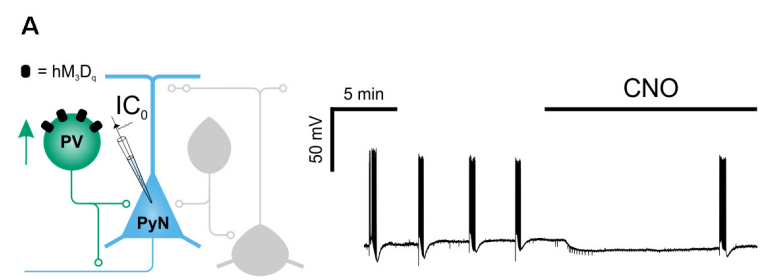

C
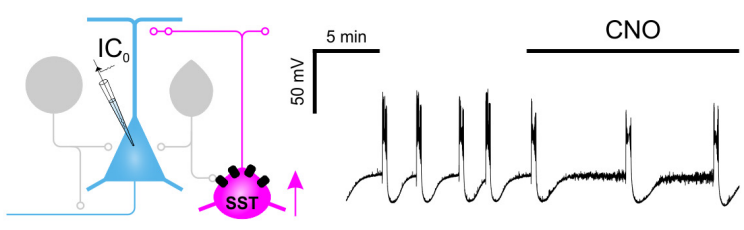

E
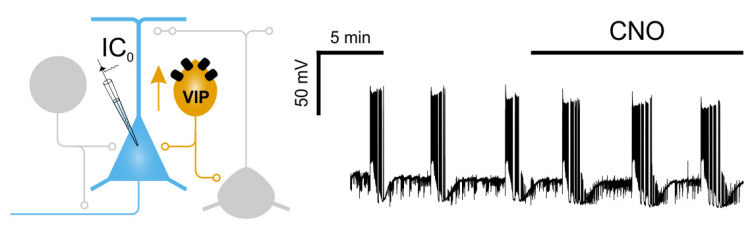

G
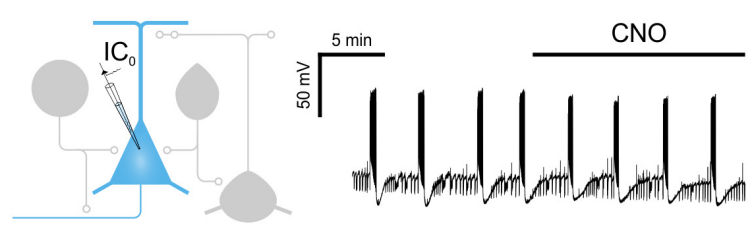
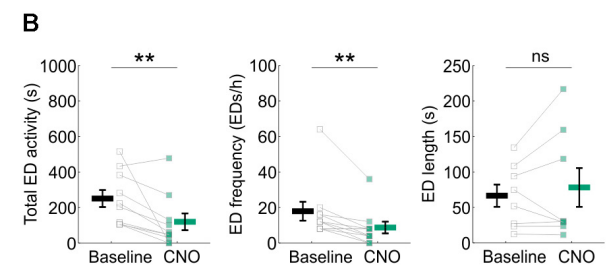

\section{D}
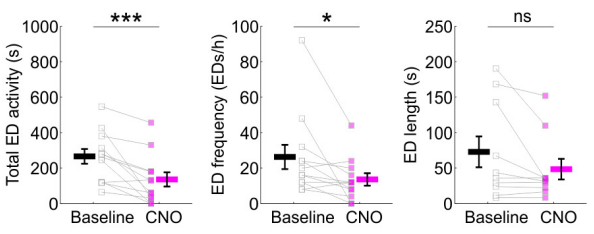

F
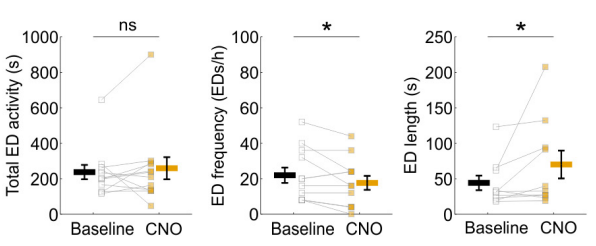

H
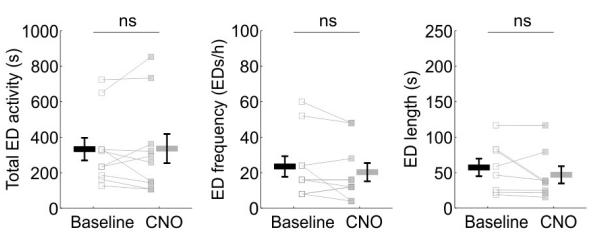

I

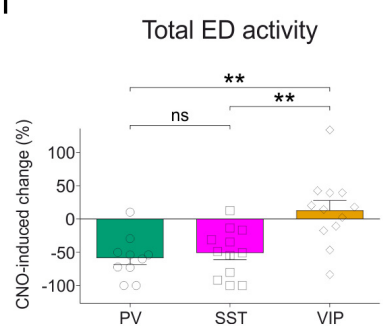

J

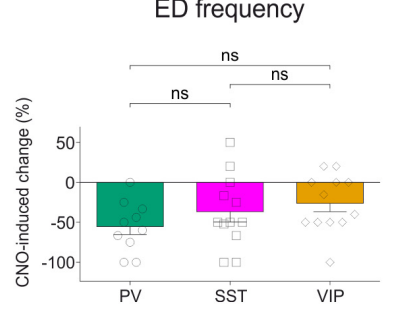

K

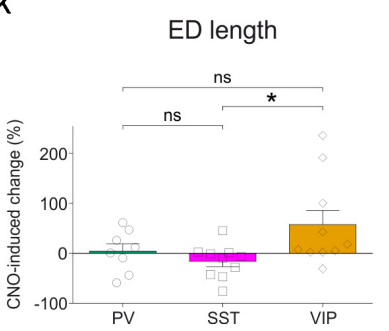

FIGURE 4 | Chemogenetic enhancement of specific GABAergic interneuron populations attenuates hippocampal EDs. (A) PV-cre mice and floxed viral constructs were used to target $\mathrm{hM}_{3} \mathrm{D}_{\mathrm{q}}$ receptors to $\mathrm{PV}$ interneurons in organotypic hippocampal brain slices. The effects of $\mathrm{CNO}$ upon spontaneous EDs were monitored by current-clamp recordings from CA1 or CA3 pyramidal neurons. (B) Population data from experiments targeting PV interneurons $(N=10$ slices) showed a reduction in total ED activity [left, $W_{(10)}=1, p=0.0039$, two-tailed Wilcoxon signed-rank test], a decrease in ED frequency [middle, $W_{(9)}=0, p=0.0039$, two-tailed Wilcoxon signed-rank test], and no change in ED length [right, $W_{(8)}=12, p=0.4609$, two-tailed Wilcoxon signed-rank test] following addition of CNO. (C) SST-cre mice were used to target $\mathrm{hM}_{3} \mathrm{D}_{q}$ receptors to SST interneurons. (D) Population data from experiments targeting SST interneurons ( $N=12$ slices) showed a reduction in total ED activity [left, $W_{(12)}=1, p=0.001$, two-tailed Wilcoxon signed-rank test], a decrease in ED frequency [middle, $W_{(12)}=6, p=0.0166$, two-tailed Wilcoxon signed-rank test], and no change in ED length [right, $W_{(10)}=10, p=0.0840$, two-tailed Wilcoxon signed-rank test] following addition of CNO. (E) VIP-cre mice were used to target $\mathrm{hM}_{3} \mathrm{D}_{\mathrm{q}}$ receptors to VIP interneurons. (F) Population data from experiments targeting VIP interneurons $(N=12$ slices) showed no change in total ED activity [left, $W_{(12)}=25, p=0.3013$, two-tailed Wilcoxon signed-rank test], a reduction in ED frequency [middle, $t_{(11)}=2.24, p=0.0468$, two-tailed paired $t$-test], and an increase in ED length [right, $W_{(10)}=6, p=0.0273$, two-tailed Wilcoxon signed-rank test] following addition of CNO. (G) Control experiments were conducted on slices that had not received floxed DREADD constructs. (H) Population data $(N=10$ slices $)$ demonstrated no change in total ED activity [left, $t_{(9)}=-0.08$, $p=0.9337$, two-tailed paired $t$-test], ED frequency [middle, $t_{(9)}=1.31, p=0.2229$, two-tailed paired $t$-test], or ED length [right, $W_{(8)}=6, p=0.1094$, two-tailed Wilcoxon signed-rank test] following the addition of CNO to control slices. (I) Total ED activity was significantly reduced by chemogenetically enhancing either PV or SST interneurons, compared to enhancing VIP interneurons $\left[F_{(2,31)}=9.747, p=0.0005\right.$, one-way ANOVA, followed by Tukey's post hoc multiple comparison tests; VIP vs. PV, $p=0.0013$; VIP vs. SST, $p=0.0025]$. (J) The frequency of EDs was reduced when PV interneurons, SST interneurons or VIP interneurons were targeted. No significant difference in the reduction of ED frequency was detected across the three interneurons $\left[F_{(2,31)}=1.583, p=0.2215\right.$, one-way ANOVA]. (K) Individual EDs became significantly longer when VIP interneurons were recruited, compared to SST interneurons $\left[F_{(2,25)}=3.772, p=0.037\right.$, one-way ANOVA, followed by Tukey's post hoc multiple comparison tests; VIP vs. SST, $p=0.0337] .{ }^{*} p<0.05,{ }^{* *} p<0.01$. 

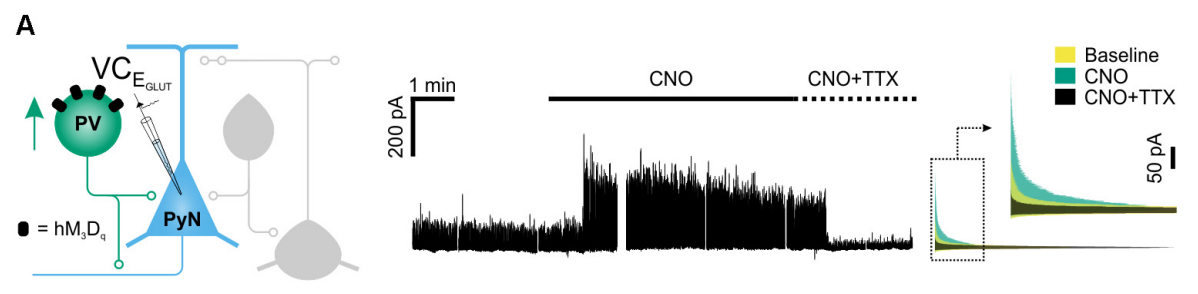

C
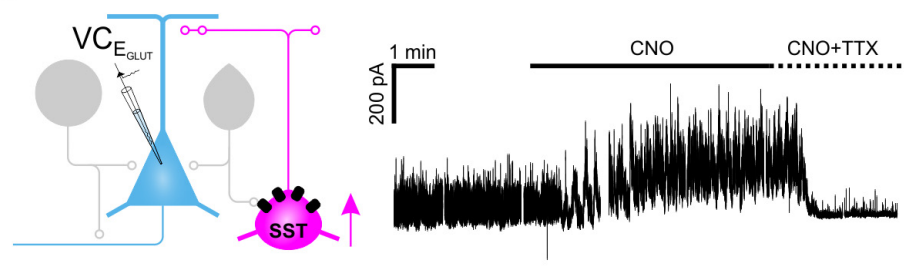

E
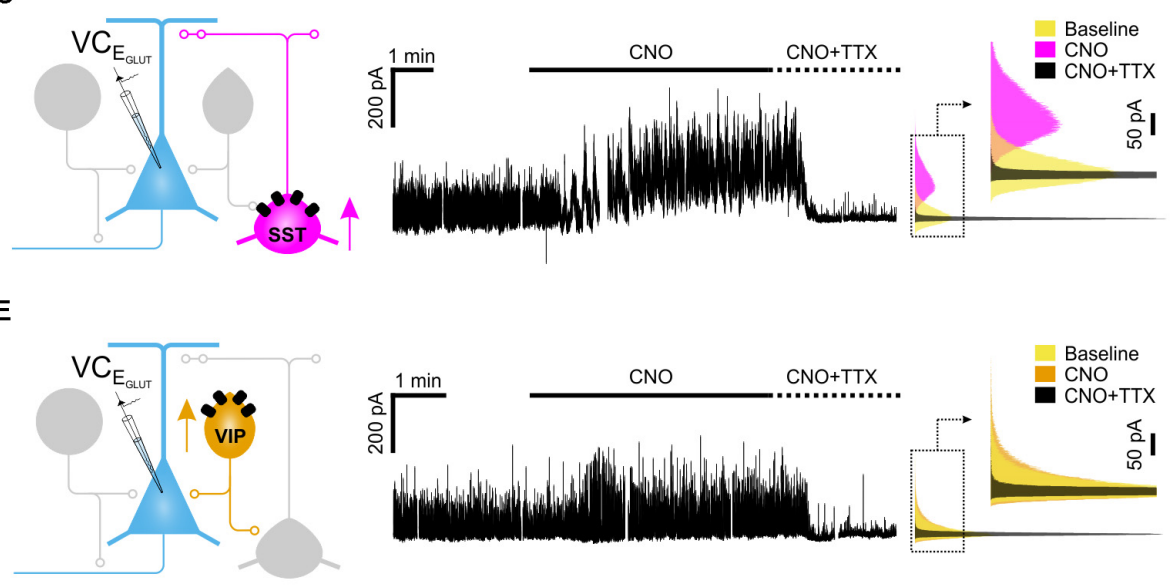

B

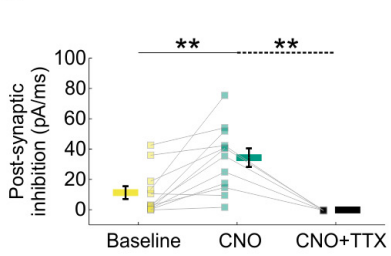

D

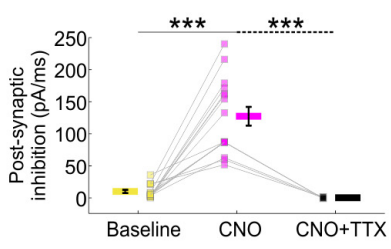

$\mathbf{F}$

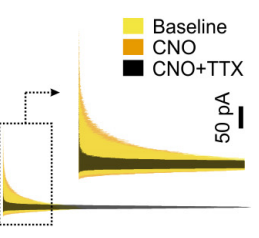

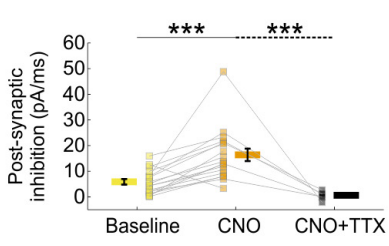

G

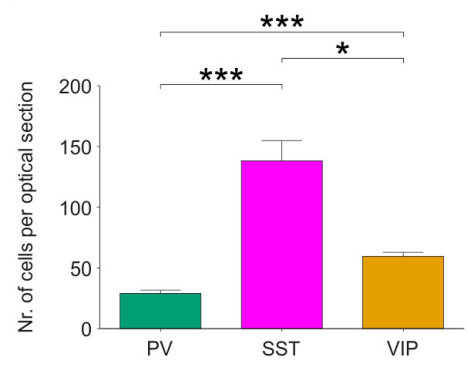

H

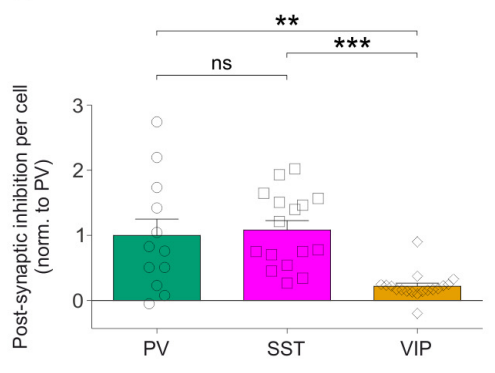

I

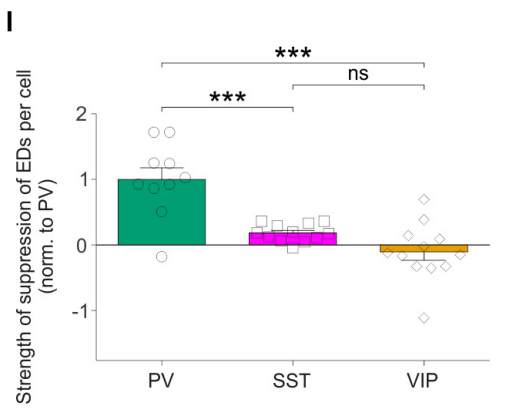

FIGURE 5 | Chemogenetic recruitment of interneuron populations generates different amounts of post-synaptic inhibition in pyramidal neurons. (A) $h M_{3} D_{q}$ receptors were targeted to PV interneurons and voltage-clamp recordings (at $\mathrm{E}_{\mathrm{GLUT}}$ ) were performed from pyramidal neurons (left). CNO application elicited a pronounced increase in inhibitory post-synaptic currents converging upon the pyramidal neuron (middle). This increase was associated with spiking activity as it was abolished by bath application of TTX. Overlapping histograms (right) illustrate the probability distribution functions for the inhibitory post-synaptic currents during baseline, CNO, and co-administration of CNO plus TTX. (B) PV recruitment resulted in a significant change in total post-synaptic inhibitory charge converging onto pyramidal neurons $\left[F_{(2,26)}=9.541, p=0.0008\right.$, one-way ANOVA, followed by post hoc Sidak's multiple comparisons tests; baseline vs. CNO, $N=12, p=0.0047 ; \mathrm{CNO}$ vs. CNO plus TTX, $N=5, p=0.0013]$. (C) $\mathrm{hM}_{3} \mathrm{D}_{\mathrm{q}}$ receptors were targeted to SST interneurons and all conventions are the same as in (A). (D) SST recruitment resulted in a significant change in total post-synaptic inhibitory charge converging onto pyramidal neurons $\left[\chi^{2}{ }_{(2)}=29.77, p<0.0001\right.$, Kruskal-Wallis test, followed by post hoc Dunn's multiple comparisons tests; baseline vs. CNO, $N=16, p<0.0001$; CNO vs. co-administration of CNO plus TTX, $N=5, p<0.0001]$. (E) hM $\mathrm{h}_{3} \mathrm{D}_{\mathrm{q}}$ receptors were targeted to VIP interneurons and all conventions are the same as in (A). (F) VIP recruitment resulted in a significant change in total post-synaptic inhibitory charge converging onto pyramidal neurons $\left[F_{(2,39)}=14.09, p<0.0001\right.$ by one-way ANOVA, followed by post hoc Sidak's multiple comparisons tests; baseline vs. CNO, $N=18, p=0.0003$; $C N O$ vs. co-administration of $C N O$ plus $T \mathrm{X}, N=6, p=0.0001]$. (G) The average number of $h M_{3} \mathrm{D}_{q}$ expressing interneurons (per $24 \mu \mathrm{m}$ optical section) differs significantly between the three interneuron populations, with an average of $29.1 \pm 2.7$ PV cells, $138.1 \pm 16.8$ SST interneurons, and $59.7 \pm 3.2$ VIP cells $\left[x^{2}{ }_{(2)}=54.44, p<0.0001\right.$, Kruskal-Wallis test, followed by Dunn's multiple post hoc comparisons; PV vs. SST, $p<0.0001 ;$ PV vs. VIP, $p<0.0001$; SST vs. VIP, $p=0.0118]$. (H) Normalizing by the size of each interneuron population (i.e., number of $\mathrm{hM}_{3} \mathrm{D}_{\mathrm{q}}$-expressing cells per slice), PV and SST interneurons were associated with similar amounts of post-synaptic inhibition, and both were significantly greater than that associated with VIP interneurons $\left[\chi^{2}{ }^{2}(2)=20.46, p<0.0001\right.$, Kruskal-Wallis test, followed by Dunn's multiple post hoc comparisons; PV vs. SST, $p>0.9999 ;$ PV vs. VIP, $p=0.0064 ;$ SST vs. VIP, $p<0.0001]$. (I) Normalizing by the size of each interneuron population, PV interneurons had the greatest effect upon the total ED activity (shown in Figure 4I) $\left[F_{(2,31)}=21.03, p<0.0001\right.$, one-way ANOVA, followed by post hoc Bonferroni's multiple comparisons tests; PV vs. SST, $p=0.0002 ; \mathrm{PV}$ vs. VIP, $p<0.0001 ;$ SST Vs. VIP, $p=0.2808] .{ }^{*} p<0.05,{ }^{* *} p<0.01,{ }^{* * *} p<0.001$ 
depths in the ventral and dorsal hippocampus, which resulted in extensive expression of the $\mathrm{hM}_{3} \mathrm{D}_{\mathrm{q}}$ receptor across the rostro-caudal axis (Figures $6 \mathrm{~B}, \mathrm{C}$ ). The majority of all virally transduced neurons were immunopositive for PV $(86.42 \pm 1.43 \%$ specificity) and the majority of PV immunopositive neurons expressed $\mathrm{hM}_{3} \mathrm{D}_{\mathrm{q}}$-mCherry $\quad(89.17 \pm 2.12 \%$ efficiency; Figure 6D).

To investigate the effect of activating PV interneurons on seizure activity in vivo, acute seizures were triggered by local application of 4-AP to one of the hippocampi, which allows for a controlled focal induction of seizure activity (Salam et al., 2017). Local application of 4-AP likely disrupted the activity of both pyramidal cells and interneurons in a limited brain area and thus generated focal seizures at the injection site, which then spread leading to generalized convulsions. This resembles the clinical situation where focal seizures are frequently initiated in an area of limited abnormal brain tissue from where they spread and propagate through normal brain networks. Such models of acutely triggered focal seizures have been important in the development of anti-epileptic drugs (Kupferberg, 2001) and allow for investigating seizure propagation and spread regardless of the initial cause of seizure initiation. Animals were randomized to receive an i.p. injection of either $\mathrm{CNO}$ or vehicle in their first experiment, and then alternated between $\mathrm{CNO}$ and vehicle for subsequent experiments. Each i.p. injection was delivered $15 \mathrm{~min}$ before the animal was placed in an arena, and their freely moving behavior was monitored for a period of $80 \mathrm{~min}$ using high-speed, high-definition cameras (Figure 7A). Following a 20-min baseline period, 4-AP was infused directly into the hippocampus according to a spaced delivery protocol (three 4-AP infusions, each separated by $12 \mathrm{~min}$; see "Materials and Methods" section).

To provide a detailed description of seizure activity and seizure spread, each animal's behavior was scored blindly using the five-point Racine scale (Figure 7B), at a sampling frequency of $1 \mathrm{~Hz}$ across at least $70 \mathrm{~min}$ per experiment (see "Materials and Methods" section). These analyses revealed that CNOmediated recruitment of hippocampal PV interneurons caused a reduction in the frequency of convulsive behaviors across the Racine 4 and 5 categories (Figure 7C). To characterize the temporal nature of these effects, the Racine scoring scale was used to generate an integrated measure of convulsive behavior that could be tracked over time (see "Materials and Methods" section). This integrated measure revealed that CNO-mediated recruitment of hippocampal PV interneurons reduced the occurrence of all convulsive behaviors by more than $80 \%$ compared to controls (Figure 7D). In contrast, CNO did not have any effect on focal seizure activity (Racine 13). Taken together, these results are consistent with the idea that increasing PV activity beyond the area affected by 4-AP reduced seizure spread and propagation. To assess whether this reduction in convulsive seizures was associated with nonspecific effects upon behavior, we monitored the animals' locomotor activity throughout the experiment. The distribution of time spent throughout the arena was indistinguishable between the vehicle and CNO-treated groups (Figures 7E,F), supporting the conclusion that the $\mathrm{CNO}$-mediated reduction in convulsive seizures was not associated with a non-specific effect upon locomotor activity. In summary our results suggest that chemogenetic recruitment of PV interneurons is effective at suppressing the spread of convulsive seizure activity in vivo.

\section{DISCUSSION}

Here, we use a combination of in vitro and in vivo studies in rodent models to demonstrate that chemogenetic enhancement of distinct populations of GABAergic interneurons can robustly reduce seizure activity. Targeted whole cell recordings from both pre-synaptic interneurons and post-synaptic principal neurons revealed that PV, SST and VIP interneuron populations all increased their firing rate and synaptic output following CNO-mediated activation of $\mathrm{hM}_{3} \mathrm{D}_{\mathrm{q}}$ DREADD receptors. Chemogenetic enhancement of either the PV or SST interneurons decreased drug-resistant synchronized epileptiform activity in vitro, through a reduction in the frequency of EDs. In contrast, enhancing VIP interneuron activity did not reduce total epileptiform activity. When accounting for the relative density of cells, PV interneurons generated the strongest effect per cell in terms of their ability to suppress EDs. Finally, to confirm the potential of such an intervention strategy, chemogenetic activation of PV interneurons was shown to produce a fivefold reduction in convulsive behaviors in an in vivo model of temporal lobe seizures.

Previous work has shown that targeting pyramidal neurons with inhibitory DREADDs can mitigate seizure activity (Kätzel et al., 2014; Avaliani et al., 2016). The current study extends this by demonstrating that, although they make up a relatively small proportion of the network, chemogenetically recruiting GABAergic populations can mediate robust anti-seizure effects. Enhancing such endogenous inhibitory mechanisms may represent an attractive intervention strategy, as interneurons can exert widespread effects on the tissue and inhibitory circuits are recruited as excitatory network activity intensifies (Trevelyan et al., 2006; Derchansky et al., 2008; Schevon et al., 2012; Cammarota et al., 2013). However, given their diversity, the particular interneuron population that is targeted is likely to be important. In line with this prediction, our data show that chemogenetic enhancement of VIP interneurons did not reduce overall seizure activity, and in fact increased the duration of individual EDs. This is in accordance with observations that the preferential post-synaptic targets of VIP interneurons are other GABAergic interneurons (Acsády et al., 1996), and that activating VIP interneurons may prevent downstream GABAergic interneurons from counteracting seizure-related excitation, since VIP interneurons specialize in disinhibitory control (Lee et al., 2013; Pi et al., 2013). In addition, it is possible that the activation of VIP interneurons leads to direct VIP release, which could increase excitability by enhancing NMDA receptor responses and excitatory transmission (Cunha-Reis et al., 2005; Yang et al., 2009). Consistent with these ideas, optically inhibiting VIP interneuron activity has been shown to generate anti-seizure effects (Khoshkhoo et al., 2017). 
A

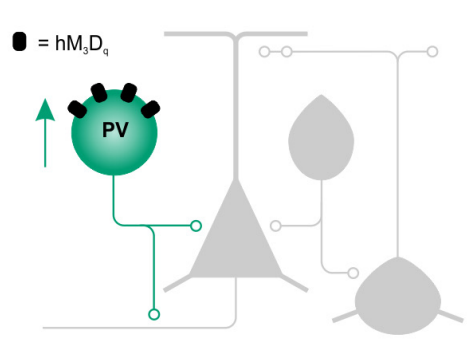

B
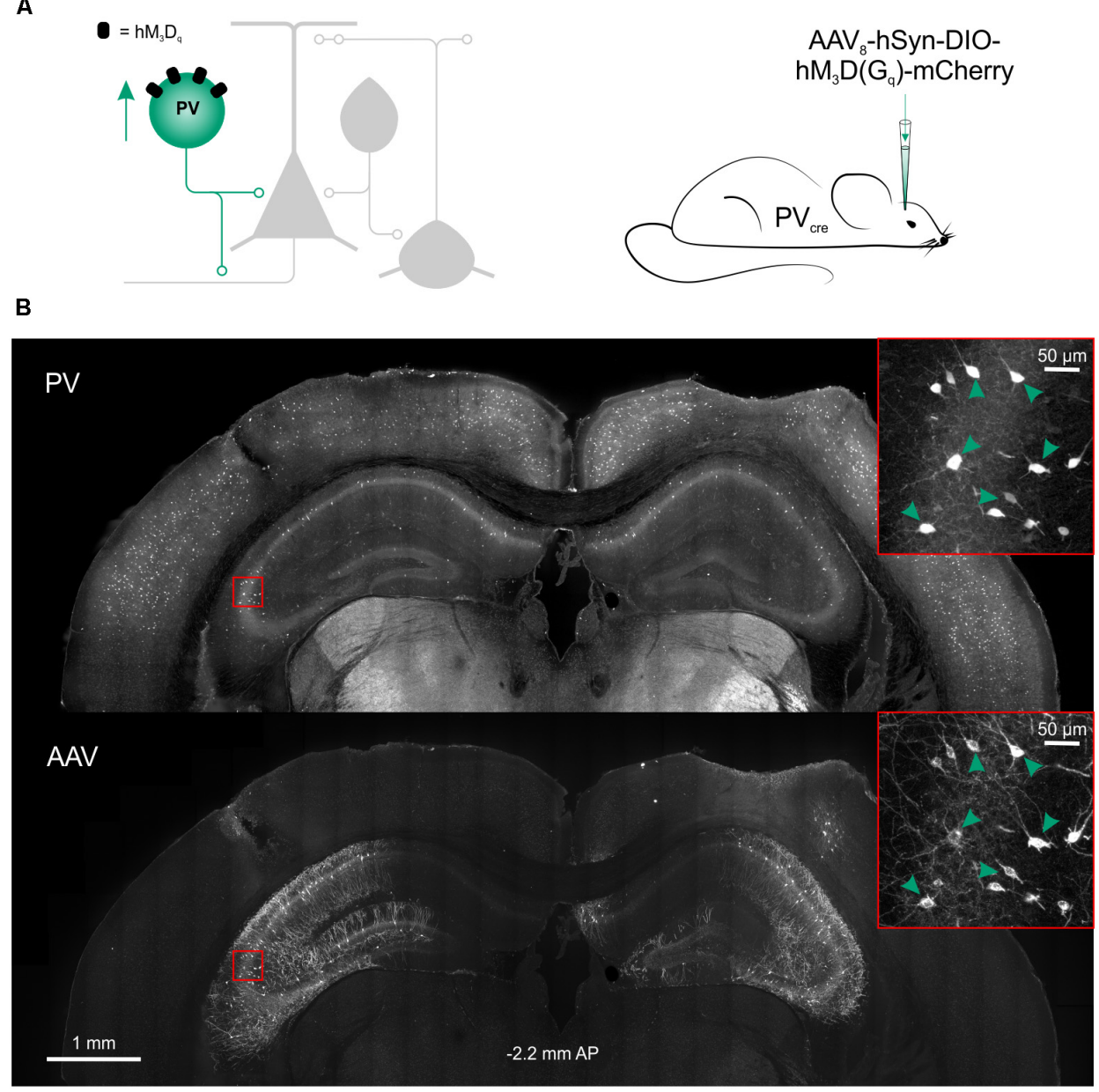

C

D
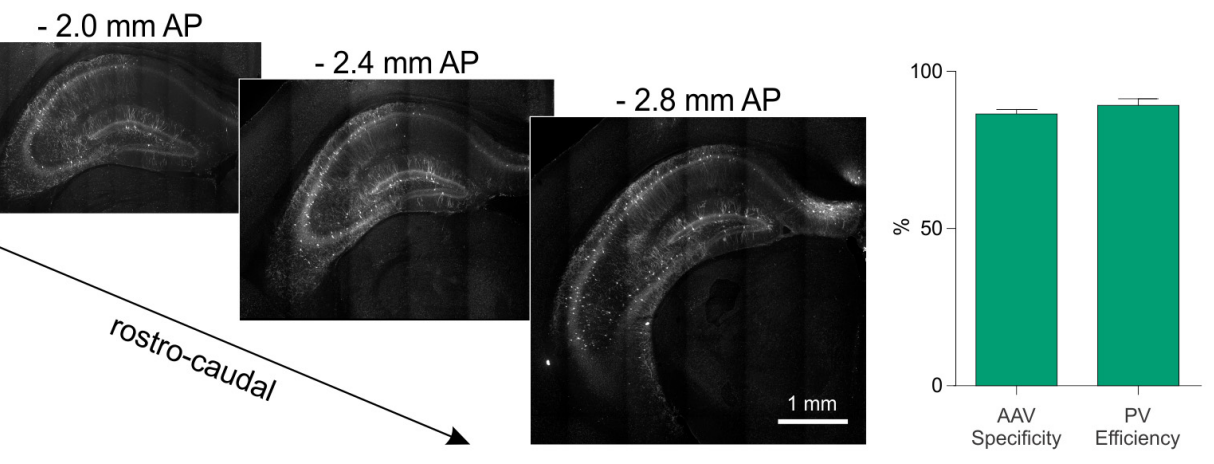

FIGURE 6 | Excitatory DREADDs can be specifically and efficiently targeted to hippocampal PV interneurons in vivo. (A) To deliver $h M_{3} D_{q}$ receptors to $P V$ interneurons in vivo (left), PV-cre mice received bilaterally injections of $\mathrm{AAV}_{8}-\mathrm{hSyn}-\mathrm{DIO}-\mathrm{hM}_{3} \mathrm{D}_{\mathrm{q}}$-mCherry into the ventral and dorsal hippocampus (right).

(B) Immunohistochemical characterization confirmed that hippocampal PV interneurons ('PV,' top) were efficiently transduced with $h M_{3} D_{q}-m C h e r r y ~(' A A V$,' bottom). Insets in red squares indicate cells co-expressing PV and $\mathrm{hM}_{3} \mathrm{D}_{\mathrm{q}}$-mCherry (arrow heads). (C) Serial sections illustrate extensive $A A V$ spread and $h M_{3} \mathrm{D}_{\mathrm{q}}$ - $\mathrm{mCherry}$ expression throughout the hippocampus. AP, anteroposterior. (D) Population data showing high targeting specificity and efficiency of the $h_{M} \mathrm{D}_{\mathrm{q}}$-mCherry expression in PV interneurons ( $N=7$ animals).

In contrast to VIP interneurons, PV and SST interneurons have been shown to preferentially target principal neurons. PV interneurons comprise mainly basket cells and axo-axonic cells, which synapse on the soma and axon-initial segment of pyramidal neurons (Klausberger et al., 2003). The dense axonal branching of $\mathrm{PV}$ interneurons is therefore restricted to the pyramidal layer, but exhibits a broad transverse extent ( $\sim 1 \mathrm{~mm}$ in rodent hippocampus). This can generate widespread 
A
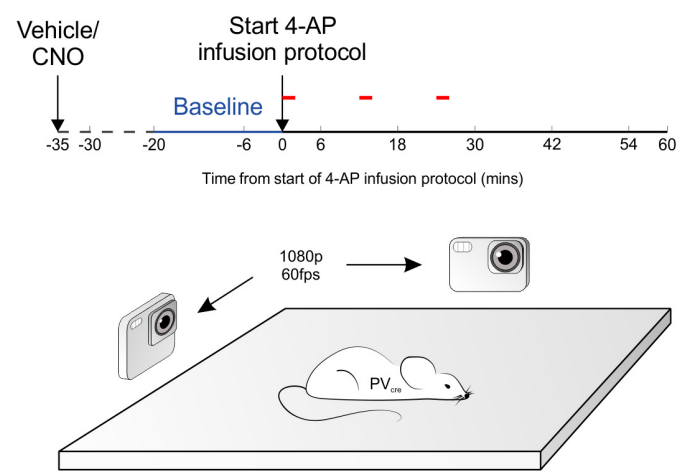

C

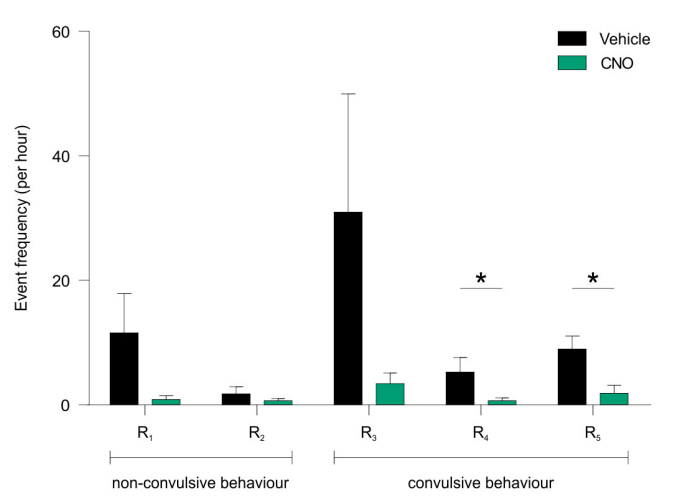

E

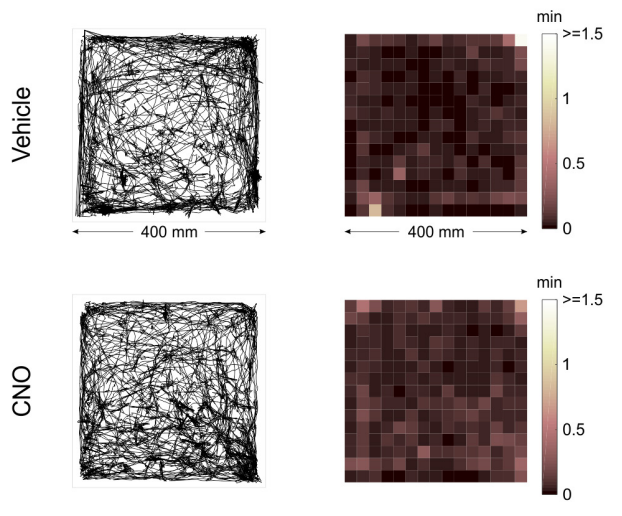

B

\begin{tabular}{|c|l|}
\hline $\begin{array}{c}\text { Racine } \\
\text { stage }\end{array}$ & Behaviours \\
\hline 1 & Orofacial clonic activity \\
\hline 2 & Head nodding \\
\hline 3 & Limb clonic activity \\
\hline 4 & Retreating/rearing with orofacial clonic activity \\
\hline 5 & Rearing and falling and/or jumping \\
\hline
\end{tabular}

D

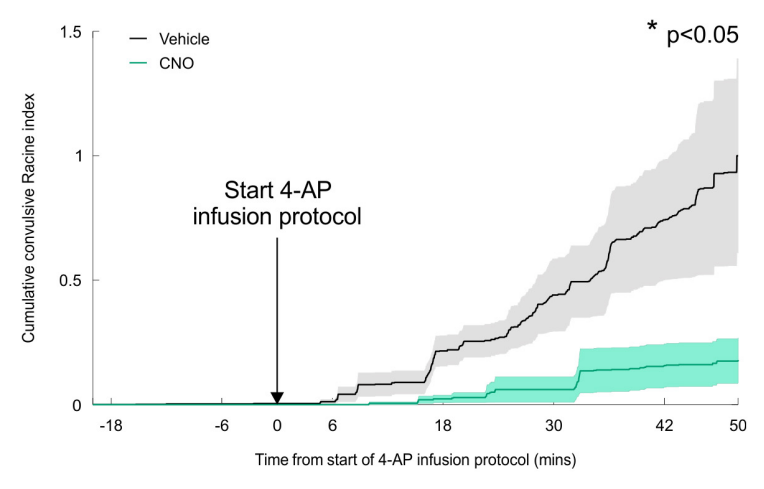

$\mathbf{F}$

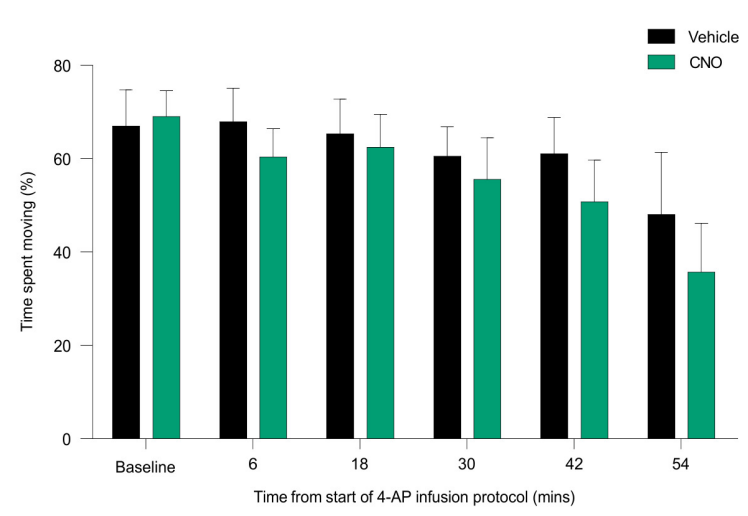

FIGURE 7 | Chemogenetic recruitment of hippocampal PV interneurons suppresses convulsive behaviors in vivo. (A) Cartoon showing experimental design for assessing seizure activity in animals expressing the $\mathrm{hM}_{3} \mathrm{D}_{\mathrm{q}}$ receptor in hippocampal PV interneurons. Fifteen minutes before behavioral monitoring, each animal received an i.p. injection of either vehicle control or CNO. Baseline behavior was then monitored for 20 min (blue period), after which the intra-hippocampal 4-AP infusion protocol was started and monitoring continued for a period of $60 \mathrm{~min}$ (red bars indicate the timing of each infusion). Behavior was recorded by two high-speed, high-definition cameras oriented at right angles to one another, while a third camera tracked the animal from above. (B) Table describing the Racine scale used to score the animal's seizure behavior from video analysis offline. (C) There was a general tendency for seizure behaviors to be suppressed by CNO across the Racine categories. The frequency of Racine 4 and 5 convulsive events was significantly reduced when CNO was administered compared to vehicle $\left[N=12\right.$ vehicle and $10 \mathrm{CNO}$ experiments; Racine $1: U_{(12,10)}=34, p=0.0571$; Racine 2: $U_{(12,10)}=59.5, p>0.9999 ;$ Racine $3: U_{(12,10)}=37, p=0.1335 ;$ Racine 4 : $U_{(12,10)}=31, p=0.0372$; Racine 5: $U_{(12,10)}=24.5, p=0.0127$, two-tailed Mann-Whitney tests]. (D) Convulsive behavior, plotted as a normalized cumulative score was significantly lower following administration of CNO compared to vehicle $\left[N=12\right.$ vehicle and 10 CNO experiments, $U_{(12,10)}=29.5, p=0.0411$, two-tailed Mann-Whitney test]. (E) For mice expressing the $\mathrm{hM}_{3} \mathrm{D}_{\mathrm{q}}$ receptor in hippocampal PV interneurons, plots illustrate tracking data and corresponding spatial distribution of time spent across the behavioral arena $(400 \mathrm{~mm}$ by $400 \mathrm{~mm}$ ). Representative data are shown for an animal receiving an i.p. injection of vehicle (top) or CNO (bottom). In each case, data is shown for a 20-min period following the start of the 4-AP infusion protocol. (F) There was no difference between the vehicle and CNO groups in terms of the percentage of time spent moving $\left[N=10\right.$ vehicle and 11 CNO experiments, treatment: $F_{(1,104)}=1.686, p=0.197$, two-way ANOVA] 
synaptic inhibition, with each cell targeting around 1,5002,000 pyramidal neurons (Freund and Buzsáki, 1996). SST interneurons meanwhile, mainly synapse on the dendrites of pyramidal neurons (Katona et al., 1999), where they regulate dendritic activation (Miles et al., 1996) and may account for as much as half of the firing rate increase following complete removal of inhibition (Lovett-Barron et al., 2012). In addition, SST interneurons can directly release SST when activated, which in turn can reduce neuronal excitability by acting via G-protein coupled receptors (Tallent and Qiu, 2008).

In our experiments, DREADD activation of either PV or SST interneurons resulted in pronounced increases in spikeevoked, post-synaptic inhibitory currents in pyramidal neurons and strong attenuation of spontaneous EDs. For both cell types, we observed a $\sim 50 \%$ reduction in the total ED activity, which was driven by a decrease in the probability of ED initiation. It has been shown that relevant concentrations of $\mathrm{CNO}$ can lead to competitive binding of some endogenous receptors (Gomez et al., 2017). However, our control experiments showing that CNO did not affect epileptiform activity in the absence of DREADD expression, support the conclusion that the antiseizure effects are mediated chemogenetically. At the population level, enhancing SST interneurons generated the largest postsynaptic inhibitory currents in pyramidal neurons. Although when adjusted for cell numbers, we estimated that individual PV interneurons elicited equivalent post-synaptic inhibitory currents and a fivefold greater attenuation of ED activity. These observations are consistent with evidence that GABAergic inputs to the axo-somatic region can exert particularly powerful inhibitory effects (Cobb et al., 1995) and that PV interneurons contribute significantly to a synaptic restraint that can oppose the initiation and propagation of seizure activity (Cammarota et al., 2013; Paz and Huguenard, 2015). In the context of epilepsy, the targeting of PV interneurons may also be more preferable because there are reports that SST interneurons become depleted (Robbins et al., 1991), whereas PV interneurons survive in epileptic animals (Sloviter, 1991; Sloviter et al., 2003) and epilepsy patients (Sloviter et al., 1991).

In agreement with these ideas and our in vitro results, we demonstrate that excitatory DREADDs in PV interneurons can generate potent anticonvulsant effects in vivo. Direct application of 4-AP to one hippocampus likely generated initial focal seizure activity by blocking $\mathrm{K}^{+}$conductances and altering the activity of both pyramidal and inhibitory neurons within local networks. Under control conditions, these focal seizures spread and progressed to full convulsions (Racine 5). However, chemogenetically increasing the activity of PV interneurons prevented the spread, as reflected by an $80 \%$ reduction in convulsive behavior. It has recently been shown that $\mathrm{CNO}$ does not cross the blood brain barrier in naive rats, but is rather metabolized to clozapine that can cross the blood brain barrier and activate DREADDs directly (Gomez et al., 2017). It therefore seems likely that the CNO-mediated effects in our in vivo studies involved the metabolism of $\mathrm{CNO}$ to clozapine, although seizure-related activity could influence the permeability of the blood brain barrier and the relative contribution of CNO. Future studies could test the potential to enhance DREADDs activity in interneurons by direct clozapine administration. Indeed, clozapine is already approved as a drug for use in humans and DREADDs activation requires very low concentrations of clozapine (Gomez et al., 2017), which may make it an attractive option for translational studies.

At the time of submission of our paper, a report was just published that showed that PV interneuron chemogenetic activation in a chronic model of epilepsy was also effective (Wang et al., 2018). The same study shows that chemogenetic inactivation of excitatory neurons resulted in impaired cognitive function. While enhancing PV interneurons may affect cognitive processes (Zou et al., 2016), we observed that reduced convulsive behavior was not associated with general changes in locomotor activity, suggesting that the effects of chemogenetically recruiting PV interneurons may become more evident when the network enters a pre-epileptic state. This indicates that chronic enhancement of interneuron activity would seem to be the more promising strategy for controlling seizure activity compared to direct silencing of excitatory neurons, which might have more serious side effects, e.g., on memory processes. The suppression of focally evoked seizures in vivo in the current study complements our results from the in vitro drug-resistant model of spontaneous EDs, demonstrating efficacy across both acute and chronic models of seizure activity. More generally, the data confirm that chemogenetically enhancing a specific interneuron population can produce effective suppression of epileptiform synchronization.

A series of studies using rodent models have successfully applied optogenetic approaches for disrupting epileptiform networks. Like chemogenetic approaches, optogenetics lends itself to cell-type targeting and this work has helped define the role of different interneuron types. For example, it has been shown that when combined with real-time seizure detection methods, temporally controlled optogenetic activation of interneuron populations can provide effective disruption of epileptic activity (Krook-Magnuson et al., 2013; Ledri et al., 2014; Sessolo et al., 2015). This work has also revealed that optogenetic activation of interneurons can actually initiate epileptiform activity, in a manner that may depend on the network state (Sessolo et al., 2015; Yekhlef et al., 2015; Assaf and Schiller, 2016; Chang et al., 2018). This phenomenon seems to be associated with the pulsed light-activation and enforced synchronization of interneuron activity, which can then synchronize the network by inducing time-locked post-inhibitory rebound spiking (Sessolo et al., 2015; Chang et al., 2018). In addition to these temporal aspects, optogenetic strategies face other challenges for disrupting seizures, including the delivery of light to structures that may be deep within the brain, or to cells that may be distributed over large regions.

Chemogenetic intervention strategies may mitigate these issues, such as the potential to modulate cellular activity on larger spatial and temporal scales. Effects from chemogenetics can be coordinated across large areas of tissue, due to the systemic delivery of the activating drug (Alexander et al., 2009). Furthermore, the fact that DREADDs are G-protein coupled receptors and act through endogenous cellular mechanisms, may 
avoid unwanted effects associated with artificial synchronization of the network (Wang et al., 2017). However, their reliance upon the cell's own intracellular machinery may limit the potential for chemogenetics. Whereas optogenetic strategies have the power to use light energy to move ions against concentration gradients, chemogenetic strategies must rely upon endogenous mechanisms, at least in their current form. Nonetheless, chemogenetics lends itself to translational approaches more readily than optogenetics. We found that activation of $\mathrm{G}_{\mathrm{q}}$ coupled receptors in PV and SST interneurons can result in sufficient activity of those neurons to dramatically reduce the probability of initiation of epileptic network activity. This has direct implications for drug discovery. As more single-cell RNA sequencing studies are published, the identification of $\mathrm{G}_{\mathrm{q}}$ coupled receptors that are enriched in PV or SST cells, but not in VIP interneurons and excitatory cells will become feasible, and the present study suggests that those G-protein-coupled receptors represent potential drug targets for temporal lobe epilepsy. Yet, there remain questions about how inert the designed drugs are for the selective activation of the DREADDs, and whether these need to be refined further (Gomez et al., 2017). Any celltargeted strategy must also consider how the contributions of particular cell types may change in epilepsy. Cell types may be lost or change their signaling at different stages of the disease (Robbins et al., 1991; Cohen et al., 2002; Wang et al., 2017), their contribution may depend on their location relative to the epileptic focus (Sessolo et al., 2015) or their position in the epileptic circuit (Paz and Huguenard, 2015), and their effects may change dynamically during an individual seizure (Ellender et al., 2014). For these reasons, multiple strategies may be required, perhaps using one strategy for pathologically affected cells within the epileptic focus and another strategy for surrounding healthier circuits. Although there are no interneuron subtypespecific promoters yet that would be small enough for AAVvector-based gene therapy, future progress in this direction would capitalize on the direct translational value of such gene-based strategies, rendering cell-targeted clinical therapies possible.

\section{REFERENCES}

Acsády, L., Görcs, T. J., and Freund, T. F. (1996). Different populations of vasoactive intestinal polypeptide-immunoreactive interneurons are specialized to control pyramidal cells or interneurons in the hippocampus. Neuroscience 73, 317-334. doi: 10.1016/0306-4522(95)00609-5

Albus, K., Wahab, A., and Heinemann, U. (2008). Standard antiepileptic drugs fail to block epileptiform activity in rat organotypic hippocampal slice cultures. $\mathrm{Br}$. J. Pharmacol. 154, 709-724. doi: 10.1038/bjp.2008.112

Alexander, G. M., Rogan, S. C., Abbas, A. I., Armbruster, B. N., Pei, Y., Allen, J. A., et al. (2009). Remote control of neuronal activity in transgenic mice expressing evolved G protein-coupled receptors. Neuron 63, 27-39. doi: 10.1016/j.neuron. 2009.06.014

Armbruster, B. N., Li, X., Pausch, M. H., Herlitze, S., and Roth, B. L. (2007). Evolving the lock to fit the key to create a family of $G$ protein-coupled receptors potently activated by an inert ligand. Proc. Natl. Acad. Sci. U.S.A. 104, 5163-5168. doi: 10.1073/pnas.0700293104

Assaf, F., and Schiller, Y. (2016). The antiepileptic and ictogenic effects of optogenetic neurostimulation of PV-expressing interneurons. J. Neurophysiol. 116, 1694-1704. doi: 10.1152/jn.00744.2015

\section{CONCLUSION}

The current work supports the use of selective chemogenetic targeting of the inhibitory system as an approach to disrupt epileptiform synchronization. Such a cell-specific pharmacological strategy has the attraction of being controllable and yet avoiding the system-wide effects of drugs that enhance GABA-mediated inhibition in a non-cell-selective fashion.

\section{AUTHOR CONTRIBUTIONS}

$\mathrm{AC}, \mathrm{AI}$, and $\mathrm{CA}$ conceived the project and wrote the manuscript. All authors contributed to the design of the experiments and reviewed the manuscript. AC and MS performed the experiments. AC analyzed the data and prepared all figures.

\section{FUNDING}

The research leading to these results has received funding from the European Research Council under the European Community's Seventh Framework Programme FP7/2007-2013, ERC Grant Agreement 617670. AC was supported by a Wellcome Trust Doctoral Fellowship [102364/Z/13/Z]. AI was supported by a Junior Research Fellowship in Medical Sciences from University College Oxford.

\section{ACKNOWLEDGMENTS}

We thank Miruna Raşcu for help in processing the mouse tracking data. We would also like to thank Professor Peter Somogyi for providing advice and the antibody against vasoactive intestinal polypeptide. An initial version of this manuscript was made available first on the bioRxiv platform at https://doi.org/10. 1101/291179.

Avaliani, N., Andersson, M., Runegaard, A. H., Woldbye, D., and Kokaia, M. (2016). DREADDs suppress seizure-like activity in a mouse model of pharmacoresistant epileptic brain tissue. Gene Ther. 23, 760-766. doi: 10.1038/ gt.2016.56

Bartos, M., and Elgueta, C. (2012). Functional characteristics of parvalbumin- and cholecystokinin-expressing basket cells. J. Physiol. 590, 669-681. doi: 10.1113/ jphysiol.2011.226175

Bergstrom, R. A., Choi, J. H., Manduca, A., Shin, H.-S., Worrell, G. A., and Howe, C. L. (2013). Automated identification of multiple seizure-related and interictal epileptiform event types in the EEG of mice. Sci. Rep. 3:1483. doi: 10.1038/ srep01483

Cammarota, M., Losi, G., Chiavegato, A., Zonta, M., and Carmignoto, G. (2013). Fast spiking interneuron control of seizure propagation in a cortical slice model of focal epilepsy. J. Physiol. 591, 807-822. doi: 10.1113/jphysiol.2012. 238154

Chamberland, S., Salesse, C., Topolnik, D., and Topolnik, L. (2010). Synapsespecific inhibitory control of hippocampal feedback inhibitory circuit. Front. Cell Neurosci. 4:130. doi: 10.3389/fncel.2010.00130

Chang, M., Dian, J. A., Dufour, S., Wang, L., Moradi Chameh, H., Ramani, M., et al. (2018). Brief activation of GABAergic interneurons initiates the transition 
to ictal events through post-inhibitory rebound excitation. Neurobiol. Dis. 109, 102-116. doi: 10.1016/j.nbd.2017.10.007

Chen, C.-C., Lu, J., Yang, R., Ding, J. B., and Zuo, Y. (2017). Selective activation of parvalbumin interneurons prevents stress-induced synapse loss and perceptual defects. Mol. Psychiatry doi: 10.1038/mp.2017.159 [Epub ahead of print].

Cobb, S. R., Buhl, E. H., Halasy, K., Paulsen, O., and Somogyi, P. (1995). Synchronization of neuronal activity in hippocampus by individual GABAergic interneurons. Nature 378, 75-78. doi: 10.1038/378 $075 \mathrm{a} 0$

Cohen, I., Navarro, V., Clemenceau, S., Baulac, M., and Miles, R. (2002). On the origin of interictal activity in human temporal lobe epilepsy in vitro. Science 298, 1418-1421. doi: 10.1126/science.1076510

Cristo, G. D., Wu, C., Chattopadhyaya, B., Ango, F., Knott, G., Welker, E., et al. (2004). Subcellular domain-restricted GABAergic innervation in primary visual cortex in the absence of sensory and thalamic inputs. Nat. Neurosci. 7, 1184-1186. doi: 10.1038/nn1334

Cunha-Reis, D., Ribeiro, J. A., and Sebastião, A. M. (2005). VIP enhances synaptic transmission to hippocampal CA1 pyramidal cells through activation of both VPAC1 and VPAC2 receptors. Brain Res. 1049, 52-60. doi: 10.1016/j.brainres. 2005.04.077

Czapiński, P., Blaszczyk, B., and Czuczwar, S. J. (2005). Mechanisms of action of antiepileptic drugs. Curr. Top. Med. Chem. 5, 3-14. doi: 10.2174/ 1568026053386962

De Simoni, A., Griesinger, C. B., and Edwards, F. A. (2003). Development of rat CA1 neurones in acute versus organotypic slices: role of experience in synaptic morphology and activity. J. Physiol. 550, 135-147. doi: 10.1113/jphysiol.2003. 039099

Derchansky, M., Jahromi, S. S., Mamani, M., Shin, D. S., Sik, A., and Carlen, P. L. (2008). Transition to seizures in the isolated immature mouse hippocampus: a switch from dominant phasic inhibition to dominant phasic excitation. J. Physiol. 586, 477-494. doi: 10.1113/jphysiol.2007.143065

Dyhrfjeld-Johnsen, J., Berdichevsky, Y., Swiercz, W., Sabolek, H., and Staley, K. J. (2010). Interictal spikes precede ictal discharges in an organotypic hippocampal slice culture model of epileptogenesis. J. Clin. Neurophysiol. 27, 418-424. doi: 10.1097/WNP.0b013e3181fe0709

Ellender, T. J., Raimondo, J. V., Irkle, A., Lamsa, K. P., and Akerman, C. J. (2014). Excitatory effects of parvalbumin-expressing interneurons maintain hippocampal epileptiform activity via synchronous afterdischarges. J. Neurosci. 34, 15208-15222. doi: 10.1523/JNEUROSCI.1747-14.2014

Freund, T. F., and Buzsáki, G. (1996). Interneurons of the hippocampus. Hippocampus 6, 347-470. doi: 10.1002/(SICI)1098-10631 9966:4<347::AID-HIPO1<3.0.CO;2-I

Gomez, J. L., Bonaventura, J., Lesniak, W., Mathews, W. B., Sysa-Shah, P., Rodriguez, L. A., et al. (2017). Chemogenetics revealed: DREADD occupancy and activation via converted clozapine. Science 357, 503-507. doi: 10.1126/ science.aan 2475

Hamm, J. P., and Yuste, R. (2016). Somatostatin interneurons control a key component of mismatch negativity in the mouse visual cortex. Cell Rep. 16, 597-604. doi: 10.1016/j.celrep.2016.06.037

Huberfeld, G., Wittner, L., Clemenceau, S., Baulac, M., Kaila, K., Miles, R., et al. (2007). Perturbed chloride homeostasis and GABAergic signaling in human temporal lobe epilepsy. J. Neurosci. 27, 9866-9873. doi: 10.1523/JNEUROSCI.2761-07.2007

Katona, I., Acsády, L., and Freund, T. F. (1999). Postsynaptic targets of somatostatin-immunoreactive interneurons in the rat hippocampus. Neuroscience 88, 37-55. doi: 10.1016/S0306-4522(98)00302-9

Kätzel, D., Nicholson, E., Schorge, S., Walker, M. C., and Kullmann, D. M. (2014). Chemical-genetic attenuation of focal neocortical seizures. Nat. Commun. 5:3847. doi: $10.1038 /$ ncomms 4847

Khoshkhoo, S., Vogt, D., and Sohal, V. S. (2017). Dynamic, cell-type-specific roles for GABAergic interneurons in a mouse model of optogenetically inducible seizures. Neuron 93, 291-298. doi: 10.1016/j.neuron.2016.11.043

Klausberger, T., Magill, P. J., Márton, L. F., Roberts, J. D. B., Cobden, P. M., Buzsáki, G., et al. (2003). Brain-state- and cell-type-specific firing of hippocampal interneurons in vivo. Nature 421, 844-848. doi: 10.1038/ nature 01374

Köhler, C. (1982). Distribution and morphology of vasoactive intestinal polypeptide-like immunoreactive neurons in regio superior of the rat hippocampal formation. Neurosci. Lett. 33, 265-270. doi: 10.1016/0304-3940(82)90382-2

Krook-Magnuson, E., Armstrong, C., Oijala, M., and Soltesz, I. (2013). On-demand optogenetic control of spontaneous seizures in temporal lobe epilepsy. Nat. Commun. 4:1376. doi: 10.1038/ncomms2376

Kupferberg, H. (2001). Animal models used in the screening of antiepileptic drugs. Epilepsia 42, 7-12. doi: 10.1111/j.1528-1167.2001.00002.x

Ledri, M., Madsen, M. G., Nikitidou, L., Kirik, D., and Kokaia, M. (2014). Global optogenetic activation of inhibitory interneurons during epileptiform activity. J. Neurosci. 34, 3364-3377. doi: 10.1523/JNEUROSCI.2734-13.2014

Lee, S., Kruglikov, I., Huang, Z. J., Fishell, G., and Rudy, B. (2013). A disinhibitory circuit mediates motor integration in the somatosensory cortex. Nat. Neurosci. 16, 1662-1670. doi: 10.1038/nn.3544

Léránth, C., Frotscher, M., Tömböl, T., and Palkovits, M. (1984). Ultrastructure and synaptic connections of vasoactive intestinal polypeptide-like immunoreactive non-pyramidal neurons and axon terminals in the rat hippocampus. Neuroscience 12, 531-542. doi: 10.1016/0306-4522(84)90071-X

Lillis, K. P., Wang, Z., Mail, M., Zhao, G. Q., Berdichevsky, Y., Bacskai, B., et al. (2015). Evolution of network synchronization during early epileptogenesis parallels synaptic circuit alterations. J. Neurosci. 35, 9920-9934. doi: 10.1523/ JNEUROSCI.4007-14.2015

Lopes, G., Bonacchi, N., Frazão, J., Neto, J. P., Atallah, B. V., Soares, S., et al. (2015). Bonsai: an event-based framework for processing and controlling data streams. Front. Neuroinformatics 9:7. doi: 10.3389/fninf.2015.00007

Lovett-Barron, M., Turi, G. F., Kaifosh, P., Lee, P. H., Bolze, F., Sun, X.-H., et al. (2012). Regulation of neuronal input transformations by tunable dendritic inhibition. Nat. Neurosci. 15, 423-430. doi: 10.1038/nn.3024

Matas, J., Chum, O., Urban, M., and Pajdla, T. (2004). Robust wide-baseline stereo from maximally stable extremal regions. Image Vis. Comput. 22, 761-767. doi: 10.1016/j.imavis.2004.02.006

Miles, R., Tóth, K., Gulyás, A. I., Hájos, N., and Freund, T. F. (1996). Differences between somatic and dendritic inhibition in the hippocampus. Neuron 16, 815-823. doi: 10.1016/S0896-6273(00)80101-4

Mula, M. (2011). GABAergic drugs in the treatment of epilepsy: modern or outmoded? Future Med. Chem. 3, 177-182. doi: 10.4155/fmc.10.296

Nistér, D., and Stewénius, H. (2008). "Linear time maximally stable extremal regions," in Proceedings of the Computer Vision - ECCV 2008 Lecture Notes in Computer Science (Berlin: Springer), 183-196. doi: 10.1007/978-3-540-88688-4_14

Pawelzik, H., Hughes, D. I., and Thomson, A. M. (2002). Physiological and morphological diversity of immunocytochemically defined parvalbumin- and cholecystokinin-positive interneurones in CA1 of the adult rat hippocampus. J. Comp. Neurol. 443, 346-367. doi: 10.1002/cne.10118

Paz, J. T., and Huguenard, J. R. (2015). Microcircuits and their interactions in epilepsy: is the focus out of focus? Nat. Neurosci. 18, 351-359. doi: 10.1038/ nn. 3950

Pi, H.-J., Hangya, B., Kvitsiani, D., Sanders, J. I., Huang, Z. J., and Kepecs, A. (2013). Cortical interneurons that specialize in disinhibitory control. Nature 503, 521-524. doi: 10.1038/nature12676

Robbins, R. J., Brines, M. L., Kim, J. H., Adrian, T., De Lanerolle, N., Welsh, S., et al. (1991). A selective loss of somatostatin in the hippocampus of patients with temporal lobe epilepsy. Ann. Neurol. 29, 325-332. doi: 10.1002/ana.41029 0316

Salam, M. T., Montandon, G., Genov, R., Devinsky, O., Campo, M. D., and Carlen, P. L. (2017). Mortality with brainstem seizures from focal 4-aminopyridineinduced recurrent hippocampal seizures. Epilepsia 58, 1637-1644. doi: 10.1111/ epi. 13846

Schevon, C. A., Weiss, S. A., McKhann, G. Jr., Goodman, R. R., Yuste, R., Emerson, R. G., et al. (2012). Evidence of an inhibitory restraint of seizure activity in humans. Nat. Commun. 3:1060. doi: 10.1038/ncomms2056

Sessolo, M., Marcon, I., Bovetti, S., Losi, G., Cammarota, M., Ratto, G. M., et al. (2015). Parvalbumin-positive inhibitory interneurons oppose propagation but favor generation of focal epileptiform activity. J. Neurosci. 35, 9544-9557. doi: 10.1523/JNEUROSCI.5117-14.2015

Sharma, S., Puttachary, S., Thippeswamy, A., Kanthasamy, A. G., and Thippeswamy, T. (2018). Status epilepticus: behavioral and electroencephalography seizure correlates in kainate experimental models. Front. Neurol. 9:7. doi: 10.3389/fneur.2018.00007 
Sloviter, R. S. (1991). Permanently altered hippocampal structure, excitability, and inhibition after experimental status epilepticus in the rat: the "dormant basket cell" hypothesis and its possible relevance to temporal lobe epilepsy. Hippocampus 1, 41-66. doi: 10.1002/hipo.450010106

Sloviter, R. S., Sollas, A. L., Barbaro, N. M., and Laxer, K. D. (1991). Calciumbinding protein (calbindin-D28K) and parvalbumin immunocytochemistry in the normal and epileptic human hippocampus. J. Comp. Neurol. 308, 381-396. doi: 10.1002/cne.903080306

Sloviter, R. S., Zappone, C. A., Harvey, B. D., Bumanglag, A. V., Bender, R. A., and Frotscher, M. (2003). "Dormant basket cell" hypothesis revisited: relative vulnerabilities of dentate gyrus mossy cells and inhibitory interneurons after hippocampal status epilepticus in the rat. J. Comp. Neurol. 459, 44-76. doi: $10.1002 /$ cne.10630

Snodgrass, S. R. (1992). GABA and epilepsy: their complex relationship and the evolution of our understanding. J. Child Neurol. 7, 77-86. doi: 10.1177/ 088307389200700114

Stoppini, L., Buchs, P.-A., and Muller, D. (1991). A simple method for organotypic cultures of nervous tissue. J. Neurosci. Methods 37, 173-182. doi: 10.1016/0165-0270(91)90128-M

Streit, P., Thompson, S. M., and Gähwiler, B. H. (1989). Anatomical and physiological properties of GABAergic neurotransmission in organotypic slice cultures of rat hippocampus. Eur. J. Neurosci. 1, 603-615. doi: 10.1111/j.1460-9568.1989.tb00366.x

Tallent, M. K., and Qiu, C. (2008). Somatostatin: an endogenous antiepileptic. Mol. Cell. Endocrinol. 286, 96-103. doi: 10.1016/j.mce.2007. 12.004

Taniguchi, H., He, M., Wu, P., Kim, S., Paik, R., Sugino, K., et al. (2011). A resource of cre driver lines for genetic targeting of GABAergic neurons in cerebral cortex. Neuron 71, 995-1013. doi: 10.1016/j.neuron.2011.07.026

Trevelyan, A. J., Sussillo, D., Watson, B. O., and Yuste, R. (2006). Modular propagation of epileptiform activity: evidence for an inhibitory veto in neocortex. J. Neurosci. 26, 12447-12455. doi: 10.1523/JNEUROSCI.2787-06. 2006

Trevelyan, A. J., Sussillo, D., and Yuste, R. (2007). Feedforward inhibition contributes to the control of epileptiform propagation speed. J. Neurosci. 27, 3383-3387. doi: 10.1523/JNEUROSCI.0145-07.2007

Tse, K., Puttachary, S., Beamer, E., Sills, G. J., and Thippeswamy, T. (2014). Advantages of repeated low dose against single high dose of kainate in C57BL/6J mouse model of status epilepticus: behavioral and electroencephalographic studies. PLoS One 9:e96622. doi: 10.1371/journal.pone.0096622

Tyan, L., Chamberland, S., Magnin, E., Camiré, O., Francavilla, R., David, L. S., et al. (2014). Dendritic inhibition provided by interneuron-specific cells controls the firing rate and timing of the hippocampal feedback inhibitory circuitry. J. Neurosci. 34, 4534-4547. doi: 10.1523/JNEUROSCI.381313.2014

Wang, Q., Shen, F.-Y., Zou, R., Zheng, J.-J., Yu, X., and Wang, Y.-W. (2017). Ketamine-induced apoptosis in the mouse cerebral cortex follows similar characteristic of physiological apoptosis and can be regulated by neuronal activity. Mol. Brain 10:24. doi: 10.1186/s13041-017-0302-2

Wang, Y., Liang, J., Chen, L., Shen, Y., Zhao, J., Xu, C., et al. (2018). Pharmacogenetic therapeutics targeting parvalbumin neurons attenuate temporal lobe epilepsy. Neurobiol. Dis. 117, 149-160. doi: 10.1016/j.nbd.2018.06.006

Yang, K., Trepanier, C. H., Li, H., Beazely, M. A., Lerner, E. A., Jackson, M. F., et al. (2009). Vasoactive intestinal peptide acts via multiple signal pathways to regulate hippocampal NMDA receptors and synaptic transmission. Hippocampus 19, 779-789. doi: 10.1002/hipo.20559

Yekhlef, L., Breschi, G. L., Lagostena, L., Russo, G., and Taverna, S. (2015). Selective activation of parvalbumin- or somatostatin-expressing interneurons triggers epileptic seizurelike activity in mouse medial entorhinal cortex. J. Neurophysiol. 113, 1616-1630. doi: 10.1152/jn.00841.2014

Zou, D., Chen, L., Deng, D., Jiang, D., Dong, F., McSweeney, C., et al. (2016). DREADD in Parvalbumin interneurons of the dentate gyrus modulates anxiety, social interaction and memory extinction. Curr. Mol. Med. 16, 91-102. doi: $10.2174 / 1566524016666151222150024$

Conflict of Interest Statement: The authors declare that the research was conducted in the absence of any commercial or financial relationships that could be construed as a potential conflict of interest.

Copyright (C) 2018 Călin, Stancu, Zagrean, Jefferys, Ilie and Akerman. This is an open-access article distributed under the terms of the Creative Commons Attribution License (CC BY). The use, distribution or reproduction in other forums is permitted, provided the original author(s) and the copyright owner(s) are credited and that the original publication in this journal is cited, in accordance with accepted academic practice. No use, distribution or reproduction is permitted which does not comply with these terms. 